\title{
Neural crest-derived neurons are replaced by a newly identified mesodermal lineage in the post-natal and aging enteric nervous system
}

\author{
Subhash Kulkarni ${ }^{1 \#, ~ M o n a l e e ~ S a h a ~}{ }^{1}$, Laren Becker², Zhuolun Wang ${ }^{1}$, Guosheng Liu ${ }^{1}$, Jenna Leser ${ }^{1}$, \\ Mithra Kumar ${ }^{1}$, Shriya Bakhshi ${ }^{1}$, Matthew Anderson ${ }^{3}$, Mark Lewandoski ${ }^{3}$, Jared Slosberg ${ }^{4}$, \\ Sushma Nagaraj ${ }^{1}$, Elizabeth Vincent ${ }^{4}$, Loyal A. Goff ${ }^{4,5,6}$, Pankaj Jay Pasricha ${ }^{1}$
}

\begin{abstract}
Affiliations
${ }^{1}$ Center for Neurogastroenterology, Department of Medicine, Johns Hopkins University - School of Medicine, Baltimore, MD 21205

2Division of Gastroenterology, Stanford University - School of Medicine, Stanford CA 94301

${ }^{3}$ Center for Cancer Research, National Cancer Institute, Frederick, MD 21702

${ }^{4}$ Department of Genetic Medicine, Johns Hopkins University - School of Medicine, Baltimore, MD 21205

${ }^{5}$ Department of Neuroscience, Johns Hopkins University - School of Medicine, Baltimore, MD 21205

${ }^{6}$ Kavli Neurodiscovery Institute, Johns Hopkins University - School of Medicine, Baltimore, MD 21205

\#Address all correspondence to Subhash Kulkarni, Ph.D. Assistant Professor, Department of Medicine, skulkar9@jh.edu
\end{abstract}

\section{ABSTRACT}

The enteric nervous system (ENS), a collection of neurons contained in the wall of the gut, is of fundamental importance to gastrointestinal and systemic health. According to the prevailing paradigm, the ENS arises from progenitor cells migrating from the embryonic neural crest and remains largely unchanged thereafter. Here, we show that the composition of maturing ENS changes with time, with a decline in neural-crest derived neurons and their replacement by mesoderm-derived neurons. Single cell transcriptomics and immunochemical approaches establish a distinct expression profile of mesodermderived neurons. The dynamic balance between the proportions of neurons from these two different lineages in the post-natal gut is dependent on the availability of their respective trophic signals, GDNF-RET and HGF-MET. With increasing age, the mesoderm-derived neurons become the dominant form of neurons in the ENS, a change associated with significant functional effects on intestinal motility. Normal intestinal function in the adult gastrointestinal tract therefore appears to require an optimal balance between these two distinct lineages within the ENS.

\section{INTRODUCTION}

The enteric nervous system (ENS) is a large collection of neurons and related cells that resides within the gastrointestinal wall and regulates gut motility and secretion along with modulating epithelial and immune cell function (Jarret et al., 2020; Kulkarni et al., 2018). During fetal development, the mammalian ENS is populated by neurons and glia derived from neural crest (NC)-derived precursors (Anderson et al., 2006; Bergner et al., 2014; Hao and Young, 2009; Obermayr et al., 2013; Uesaka et al., 2016; Young et al., 2000; Young and Newgreen, 2001b). These precursors follow diverse migratory routes to colonize and innervate various parts of the gut before birth (Burns, 2005; Espinosa-Medina et al., 2017; Uesaka et al., 2015). It is not clear, however, that this lineage persists in its entirety in the adult gut, as indicated by the observed lack of expression of fluorescent reporter protein in a subpopulation of adult enteric neurons in NClineage-traced mice (Brokhman et al., 2019; Laranjeira et al., 2011). Alternative sources of enteric neurons that have been proposed in the literature include the ventral neural tube (VENT) (Sohal et al., 2002), or the Pdx1-expressing pancreatic endoderm (Brokhman et al., 2019), but the interpretation of these studies has been limited by the lack of robust lineage markers for non-NC derived neurons (Habeck, 2003).

Confirmation of a second, distinct lineage of enteric neurons in adults is important for our understanding of healthy gut physiology as well as the pathogenesis of acquired disorders of the ENS. In this study, we found that while the early post-natal ENS is derived from the canonical NC lineage, this pattern changes rapidly as the ENS matures, due to the arrival and continual expansion of a novel population of $\underline{\text { Mesoderm-derived Enteric Neurons }}$ (MENs) which represent an equal proportion of the ENS in young adulthood and with increasing age, eventually outnumber the $\underline{N} C$-derived Enteric $\underline{\text { Neurons }}$ (NENs). We also found that, while the NEN population is regulated by glial derived neurotrophic factor (GDNF) signaling through its receptor RET, the MEN population is regulated by hepatocyte growth factor (HGF) signaling. Normal agedependent loss of GDNF-RET signaling or its modulation by genetic and pharmacological means affected the proportion of NENs in the adult ENS. Conversely, the rising proportion of MENs with age correlated with increasing levels of HGF in the gut, a process that was accelerated by pharmacological doses of HGF.

These results indicate for the first time that the mesoderm is an important source of neurons in the second largest nervous system of the body. The increasing proportions of neurons of mesodermal lineage is a natural consequence of maturation and aging, and by implication, may be associated with acquired adult-onset disorders of the ENS. These findings therefore provide a new paradigm for understanding the structure and function of the adult ENS in health, age-related gut dysfunction and other acquired disorders of gastrointestinal motility. 


\section{BioRxiv Preprint}

A
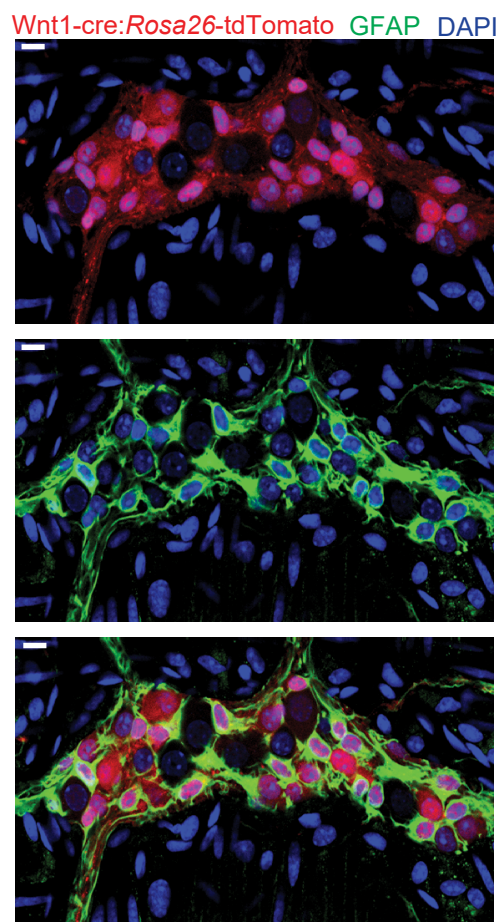

C

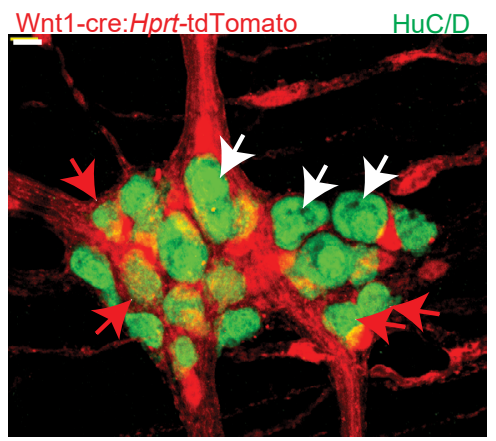

\section{RESULTS}

\section{Only half of all adult enteric neurons are derived from the neural crest}

We isolated tissues of small intestinal longitudinal muscle-myenteric plexus (LM-MP) layers from adult (postnatal day 60; P60) Wnt1-cre:Rosa26-tdTomato mice, in which tdTomato is expressed by all derivatives of Wnt $1^{+}$ migratory NC cells, including enteric neurons and enteric glia (Becker et al., 2012; Becker et al., 2011; Becker et al., 2013). In these tissues, we found that GFAP, a commonly used marker for glia, was always co-expressed with tdTomato by cells in the adult myenteric ganglia (Fig 1A). By contrast, we found that tdTomato-expression is absent in many adult myenteric neurons labeled with the pan-neuronal marker HuC/D (Fig 1B). To ensure that the lack of tdTomato expression was not due to silencing of the Rosa26 locus in these cells, we verified the fidelity of reporter expression using small intestinal LM-MP tissues from an analogous Wnt1-cre:Hprt-tdTomato lineage-traced mouse line, in which tdTomato was expressed from the
Fig 1: A subpopulation of adult myenteric neurons, but not glia, are derived from a non-neural crest lineage.

(A) Enteric glia, labeled with GFAP (green) in the myenteric plexus from an adult Wnt1-cre:Rosa26-tdTomato mouse, universally co-express tdTomato (red), indicating their origin from the neural crest. Nuclei are labeled with DAPI (blue). Scale bar $=10 \mu \mathrm{m}$.

(B-D) Interrogation of three different neural crest-specific lineage-traced mouse lines indicates that only a subpopulation of HuC/D+ adult enteric neurons (green) is derived from the neural crest, as seen by its co-expression of tdTomato (red, red arrows), driven by its respective neural crest-specific gene lineage marker, as indicated (B) Wnt1-cre:Rosa26-tdTomato; (C) Wnt1-cre:Hprt-tdTomato; (D) Pax3-cre:Rosa26-tdTomato. HuC/ $D+$ neurons (green) that do not express tdTomato (white arrows) are not derived from the neural crest. Nuclei are labeled with DAPI (blue) in (B). Scale bar $=10 \mu \mathrm{m}$.

Hprt locus in a Wnt1-cre-dependent manner (Hprt locus is X-inactivated in females, hence only adult male mice were used)(Antal et al., 2014). tdTomato reporter expression from the Hprt locus was similarly found lacking in a population of $\mathrm{HuC} / \mathrm{D}^{+}$ adult myenteric neurons (Fig $1 \mathrm{C}$, Fig $\mathrm{S} 1)$. We then tested whether these tdTomato ${ }^{-}$neurons are derived from a Wnt1- NC-lineage by using small intestinal LM-MP tissues from the Pax3-cre:Rosa26-tdTomato lineagetraced mouse line, where tdTomato driven by the Pax3-cre transgene labels the derivatives of the neural tube including the pre-migratory NC (Debbache et al., 2018; Freyer et al., 2011). Here again, we observed a lack of tdTomato reporter expression in a population of $\mathrm{HuC} / \mathrm{D}^{+}$ adult myenteric neurons (Fig 1D). A similar lack of another reporter (YFP) was observed previously in adult myenteric neurons from a Sox10-cre NC-lineage-traced mouse (Laranjeira et al., 2011). The absence of reporter expression in a population of $\mathrm{HuC} / \mathrm{D}^{+}$neurons in three different $\mathrm{NC}$ specific lineage fate mapping adult mouse lines suggests that a population of adult myenteric neurons is not derived from the NC-lineage. Importantly, in these lineage-traced mice, myenteric ganglia were found to contain tdTomato high and tdTomato ${ }^{10 w}$ neurons and due care was taken to image subsets (Fig S1). Both tdTomato ${ }^{\text {high }}$ and tdTomato ${ }^{\text {low }}$ neurons were classified as tdTomato ${ }^{+}$and only neurons that did not show any tdTomato-expression were classified as tdTomato non-expressing neurons. 
Fig 2: Lineage fate-mapping from mesoderm-specific lineage-traced mice and expression of MHCst provide evidence of the mesodermal-derivation of half of all adult enteric neurons.

(A, B). Interrogation of two mesoderm-lineage-traced mouse lines indicate that a subpopulation (white arrows) of $\mathrm{HuC} / \mathrm{D}+$ (green) adult enteric neurons is derived from the mesoderm, as seen by their co-expression of tdTomato (red) in (A) Tek-cre:Hprt-tdTomato and (B) Mesp1-cre:Rosa26-tdTomato adult male mice. Also seen are HuC/D+ neurons that do not express tdTomato (green arrows) in either mouse line, indicating their non-mesodermal, presumably neural crest origin. Nuclei are labeled with DAPI. Scale bar $=10 \mu \mathrm{m}$.

(C-E) MHCst immunolabeling exclusively marks all the mesoderm-derived adult neurons. MHCst+ (cyan) labels all tdTomato+ (red) $\mathrm{HuC} / \mathrm{D}+$ (green) adult neurons (white arrows) but not the tdTomato- neurons (yellow arrow) in the mesoderm-specific (C) Mesp1-cre:Rosa26-tdTomato and (D) Tek-cre:Rosa26-tdTomato lineage-traced lines. By contrast $(E)$ MHCst (green) exclusively labels tdTomatoadult myenteric neurons (white arrows) in the neural crest-specific Wnt1-cre:Rosa26-tdTomato lineage-traced line, but not tdTomato+ neural crest-derived cells (yellow arrows). Nuclei are stained with DAPI (blue). Scale bar = $10 \mu \mathrm{m}$.

(F) Adult myenteric ganglia are composed of equal numbers of mesoderm-derived enteric neurons (MENs) and neural crest-derived enteric neurons (NENs) at post-natal day 60. The proportions of MENs and NENs were calculated by counting tdTomato+ and tdTomato- neurons in the myenteric plexus tissues of Mesp1-cre:Rosa26-tdTomato ( $n=31$ ganglia from 3 mice) and Wnt1-cre:Rosa26-tdTomato ( $n=53$ ganglia from 5 mice) mice, respectively. Data represent mean \pm S.E.M.; unpaired t test was used for statistical analysis.
A
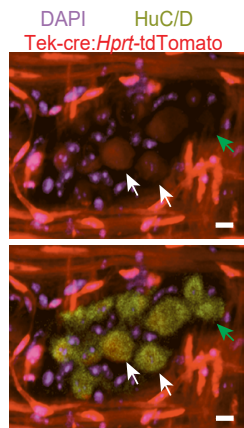

B
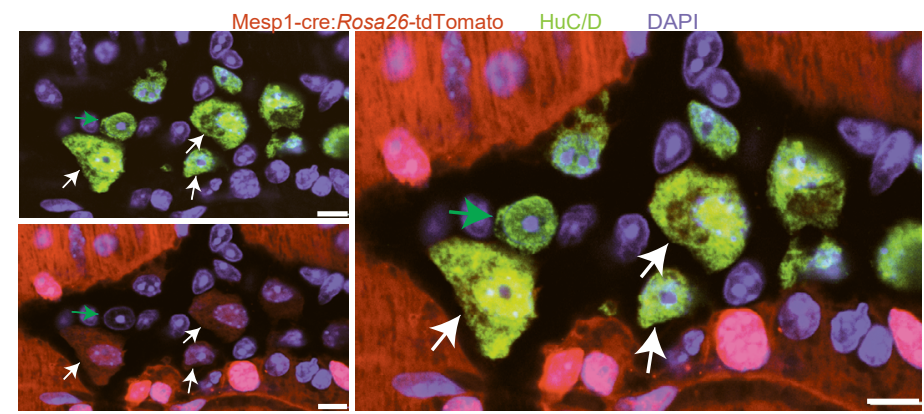

C
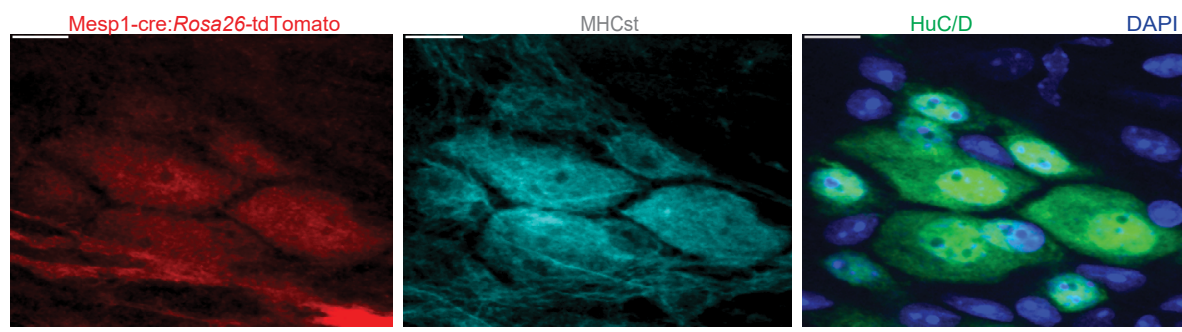

D
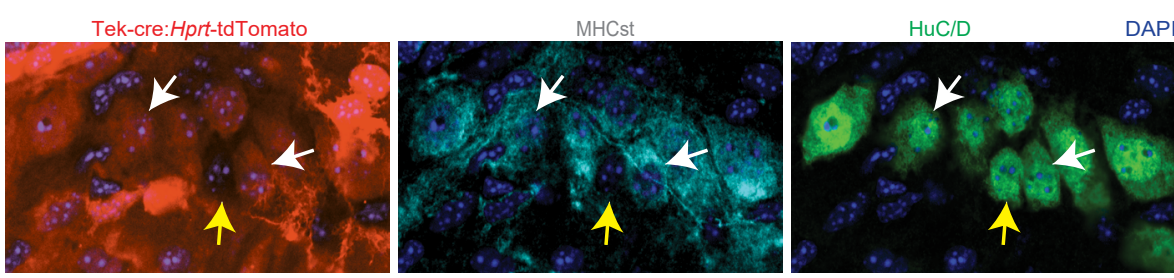

E

$\mathrm{F}$

G
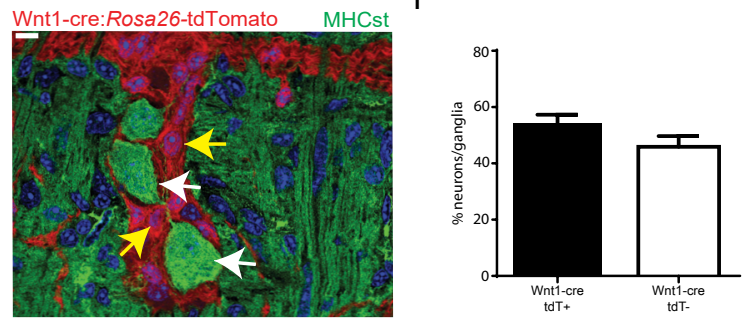

\section{Mesoderm-specific lineage fate-mapping} and marker expression confirm mesodermalderivation of the non-NC-derived adult enteric neurons

Using inducible Pdx1-cre, Foxa2-cre, and Sox17-cre transgenic mice containing floxed reporter genes, a recent report showed the presence of reporter-labeled neurons in the myenteric plexus of each of these lineage-traced mouse lines (Brokhman et al., 2019). Since these genes are expressed by derivatives of the definitive endoderm, this was inferred as evidence of endodermal derivation of these neurons. However, in a Pdx1-cre lineage-traced mouse line, many neuroectoderm-derived neurons of the central nervous system have also been previously shown to be derived from Pdx1-expressing cells (Honig et al., 2010), suggesting that Pdx1 is not an exclusive endodermal marker. Similarly, Foxa2 is expressed by mesoderm-derived cells in the heart (Bardot et al., 2017). Since Sox17 also labels mesodermderived cells, especially those in the gut wall including cells of the intestinal vasculature (Burtscher et al., 2012; Herrick and Mutsaers, 2004; Kawaguchi et al., 2007; Wilm et al., 2005), we hypothesized that the alternate developmental source of adult myenteric neurons may be the embryonic mesoderm, instead of the endoderm as suggested by Brokhman et al. Mesoderm-derived cells during murine embryogenesis are known to express endothelial cell markers such as Tek (also known as Tie2) (Ema et al., 2006). We therefore used a Tek-cre:Hprt-tdTomato lineage-traced mouse line and found that both endothelial cells and myenteric neurons expressed tdTomato (Fig 2A). Since Tek is not expressed by adult ENS cells (Fig S2), the derivation of a population of adult myenteric neurons from $\mathrm{Tek}^{+}$cells suggests a putative $\mathrm{Tek}^{+}$mesodermal lineage for these cells.

We then used Mesp1 expression, a specific marker for the embryonic mesoderm (Chan et al., 2013; Devine et al., 2014; Klotz et al., 2015; Lescroart et al., 2014), to label the lineage of non-NC derived adult enteric neurons. In the small intestinal LM-MP tissue from P60 Mesp1- 


\section{BioRxiv Preprint}

cre:Rosa26-tdTomato mice, we found that many $\mathrm{HuC} / \mathrm{D}^{+}$ myenteric neurons expressed tdTomato, indicating their derivation from the mesoderm (Fig 2B). Mesp1-cre:Rosa26tdTomato $^{+}$neurons were also labeled with S46 antibody, which labels the slow tonic myosin heavy chain protein MHCst that is expressed exclusively by mesoderm-derived cells (Plaisance et al., 2016; Sokoloff et al., 2007; Stockdale and Miller, 1987) (Fig 2C). MHCst immunostaining was exclusively observed in all Tek-derived neurons (Fig 2D), and in all Wnt1-cre:Rosa26-tdTomato non-expressing non-NC lineage neurons, providing confirmatory evidence for their mesodermal origin (Fig 2E, Fig S3A). Thus, two distinct populations- Mesp1-derived mesoderm-derived enteric neurons (MENs), and Wnt1-derived neural crestderived enteric neurons (NENs)- together account for all the adult small intestinal myenteric neurons (Fig 2F, G).

The proteins RET, a receptor tyrosine kinase that transduces GDNF signaling in NC-derived enteric neuronal precursors, and MET, a receptor for hepatocyte growth factor (HGF), are expressed by different subsets of adult myenteric neurons (Avetisyan et al., 2015). Since MET is classically expressed by mesoderm-derived cells (Rappolee et al., 1996), we tested whether expression of MET was exclusive to MENs. Using the Wnt1-cre:Rosa26-

Fig 3: MENs can be distinguished from NENs by surface marker profiles and contain different sub-populations of neuronal subtypes.

(A) RET immunostaining (green) only labels tdTomato+ neural crest-lineage cells (green arrow) in the myenteric plexus of adult Wnt1-cre:Rosa26-tdTomato mice; tdTomato- cells of MEN lineage (white arrows) do not express RET. Nuclei are labeled with DAPI (blue). Scale bar $=10 \mu \mathrm{m}$.

(B) MET immunostaining (green) exclusively labels a subpopulation of tdTomato- myenteric cells in Wnt1-cre:Rosa26-tdTomato adult mice, while the neural crest-derived tdTomato+ cells (red) do not express MET. Scale bar $=10 \mu \mathrm{m}$.

(C) MET (green) is expressed by some (white arrows) but not all (red arrow) tdTomato+ mesodermal neurons in the myenteric plexus of Mesp1-cre:Rosa26-tdTomato mice. Nuclei are labeled with DAPI (blue). Scale bar $=10$ $\mu \mathrm{m}$.

(D) NOS1 is expressed by both tdTomato+ NENs (red, white arrows) and tdTomato- MENs (blue arrows) in an adult Wnt1-cre:Rosa26-tdTomato mouse but most MENs do not express NOS1 (marked by *). Scale bar = $10 \mu \mathrm{m}$.

(E) The majority of tdTomato $(\mathrm{td} T)+$ neural crest-derived neurons express NOS1, a proportion significantly higher as compared with the tdT- MEN lineage in Wnt1-cre:Rosa26-tdTomato male mice $(n=32$ ganglia from 3 mice). Data represent mean \pm S.E.M.; unpaired t test was used for statistical analysis $\left(^{* * \star \star} \mathrm{p}<0.0001\right)$.

(F) Both tdTomato+ (red) and tdTomato- neurons in the myenteric plexus of an adult Wnt1-cre:Rosa26-tdTomato mouse (HuC/D, green) express CGRP (blue) A tdTomato- CGRP+ neuron is marked (white arrow) and expanded on the right to show a magnified version. Scale bar denotes $10 \mu \mathrm{m}$.

(G) In adult Wnt1-cre:Rosa26-tdTomato mice, tdTomato- MENs express CGRP in significantly greater proportions than do tdTomato+ NENs $(n=30$ ganglia from 3 mice). Data represent mean \pm S.E.M.; unpaired t test was used for statistical analysis. ( $\left.{ }^{\star \star \star} p<0.001\right)$.

$(\mathrm{H})$ tdTomato- MENs express the protein P-Cadherin/CDH-3 (green; white arrows) while tdTomato+ (red) NENs do not (blue arrows) in myenteric ganglia from adult Wnt1-cre:Rosa26-tdTomato mice. Nuclei are labeled with DAPI (blue). Scale bar denotes $10 \mu \mathrm{m}$.
tdTomato mice, we confirmed that RET expression is restricted to NC-cells and that MET was restricted to a sub-population of adult MENs (Fig 3A, B). We confirmed that MET was indeed specific to MENs by observing the co-localization of MET and Mesp1-cre:Rosa26-tdTomato expression (Fig $3 \mathrm{C}$ ), providing further evidence of the mesodermal derivation of MENs.

We then studied whether the two neuronal lineages differed phenotypically. To do this, we examined the expression of an important neurotransmitter-producing protein and a neuropeptide in the ENS: nitric oxide synthase 1 (NOS1) that produces nitric oxide, a critical molecule for regulating gut motility; and calcitonin gene related peptide (CGRP), a molecule expressed by murine intrinsic primary afferent neurons (IPANs), a subset of myenteric neurons that initiate the peristaltic reflex. Immunofluorescence analyses of NOS1 expression in the myenteric plexus from P60 Wnt1cre:Rosa26-tdTomato mice showed that the tdTomato ${ }^{+}$ NENs account for the majority of NOS1-expressing nitrergic neurons (Fig 3D, E). By contrast, we found that MENs account for the majority of CGRP-expressing neurons (Fig 3F, G). These results are in keeping with previous reports that show low to absent expression of the classical neurotransmitter genes, ChAT and NOS1 and abundant expression of IPAN marker CGRP by $\mathrm{MET}^{+}$cells (Avetisyan

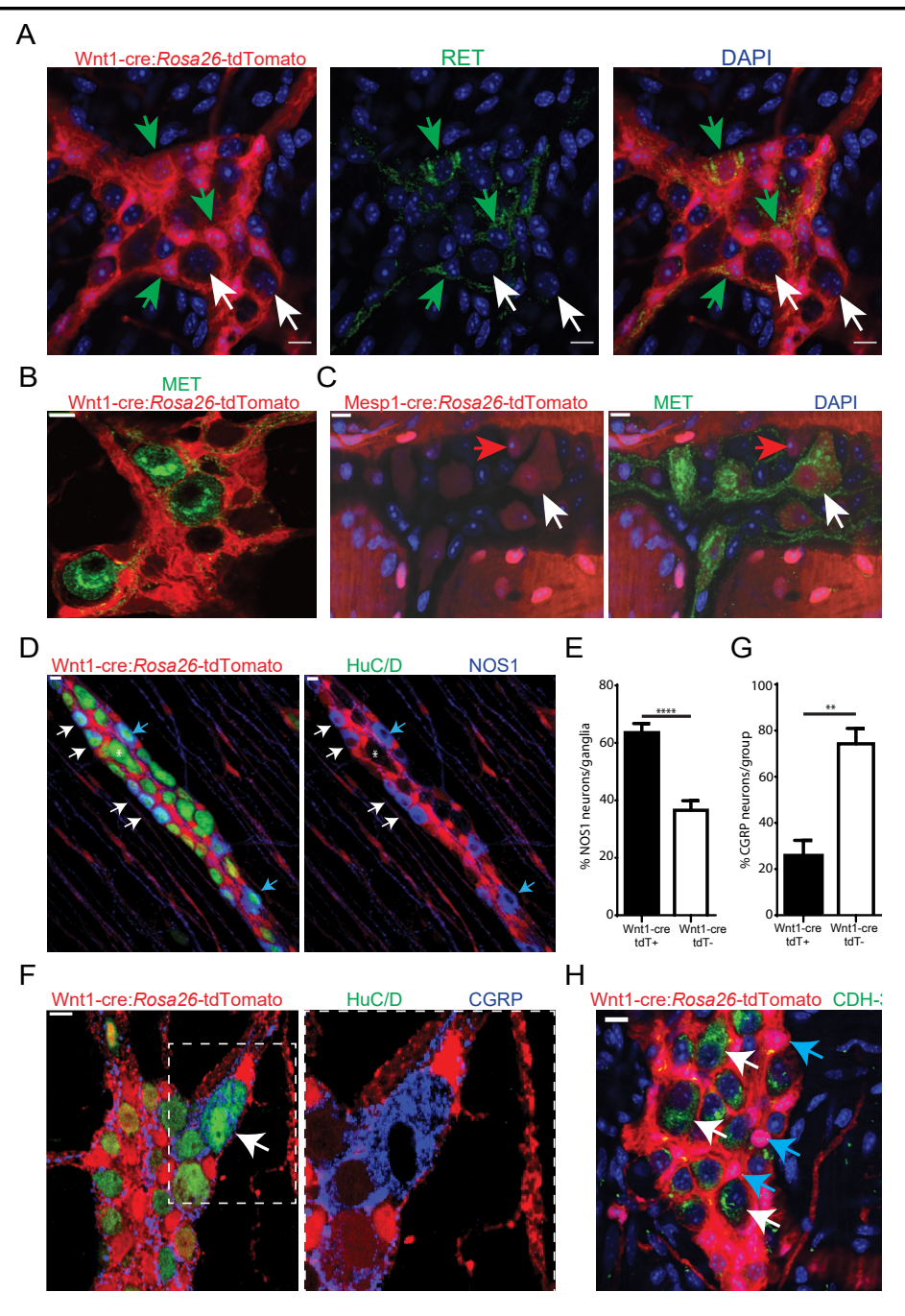


A

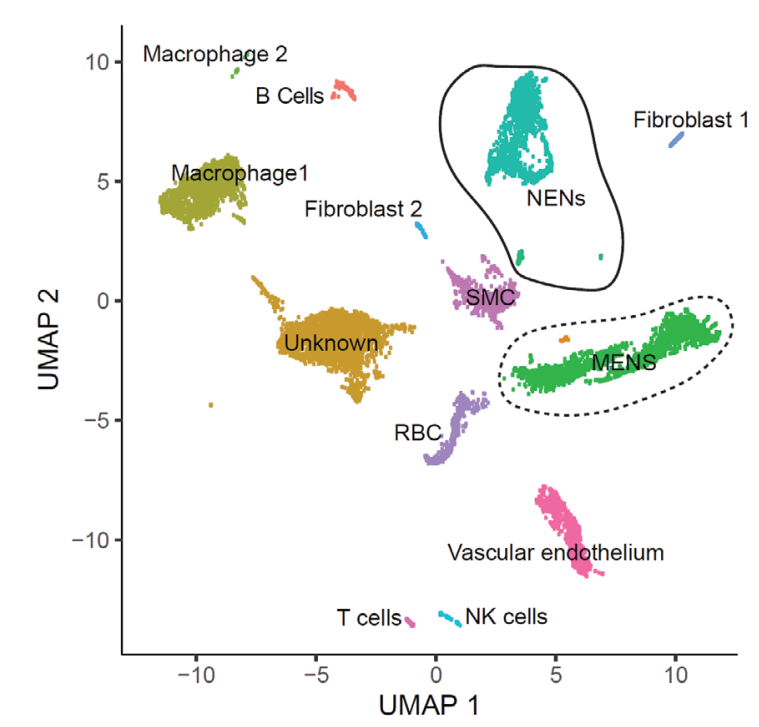

B

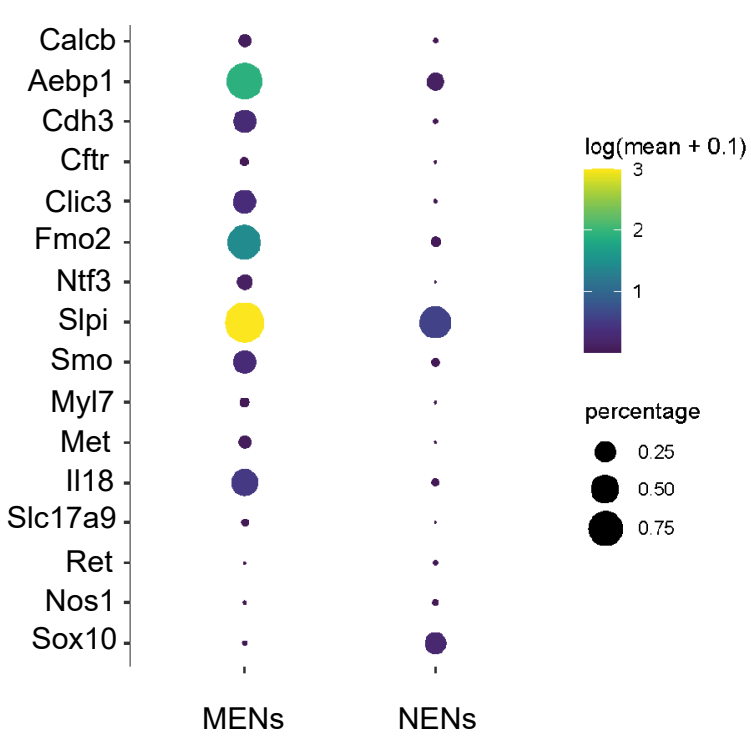

C

Wnt1-cre:Rosa26-tdTomato
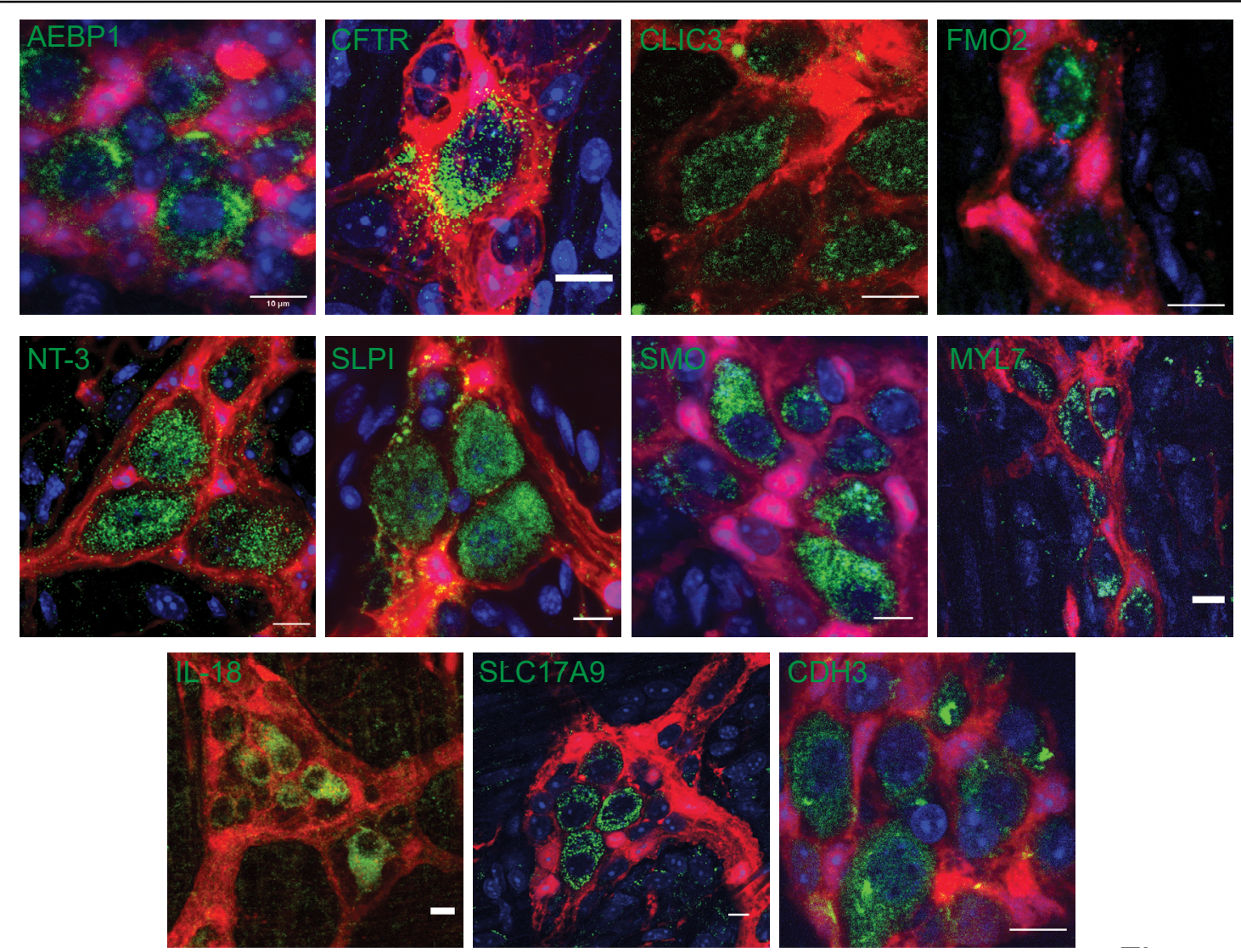

Fig 4: Single Cell RNA Sequencing (scRNAseq)-analyses identifies the distinct transcriptomic profile of the MEN cells.

(A) UMAP representation of 11,123 sequenced high-quality cells that were identified as meeting a 200 UMI minimum threshold with a mitochondrial read ratio of less than $20 \%$. Clusters were annotated by markers that were found to be cell-type specific by searching UniProt, Allen Cell Atlas and Pubmed databases. Neurons were then identified as NENs by co-expression of neural crest marker genes Ret and Sox10, or as MENs by co-expression of Calcb (CGRP), Cdh3, and Met genes.

(B) Cluster-specific expression patterns of select markers.

(C) Validation of the MENs-specific marker genes discovered in the scRNAseq analyses by immunohistochemistry and confocal microscopy of small intestinal LM-MP from adult male Wnt1-cre:Rosa26-tdTomato mice. Immunostaining of the proteins AEBP1, CFTR, CLIC3, FMO2, NT3, SLPI, SMO, MYL7, IL-18, SLC17A9, and CDH3 (green; green arrows) was found to be localized to tdTomato- MENs. tdTomato+ (red) NENs did not immunostain for these markers (red arrows). Nuclei in these tissues were labeled with DAPI (blue). Scale bar denotes $10 \mu \mathrm{m}$. 
bioRxiv preprint doi: https://doi.org/10.1101/2020.08.25.262832; this version posted August 25, 2020. The copyright holder for this preprint (which was not certified by peer review) is the author/funder. All rights reserved. No reuse allowed without permission.

\section{BioRxiv Preprint}

A Post-natal Day 11 myenteric plexus

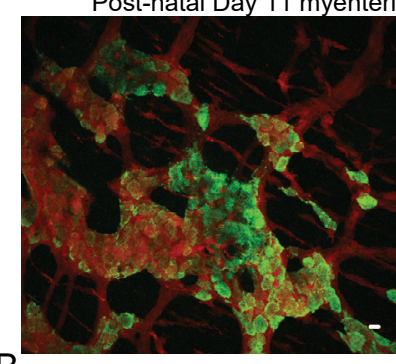

$\mathrm{B}$

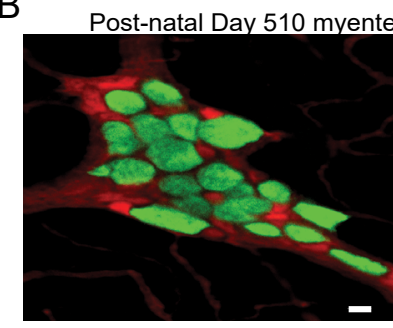

C
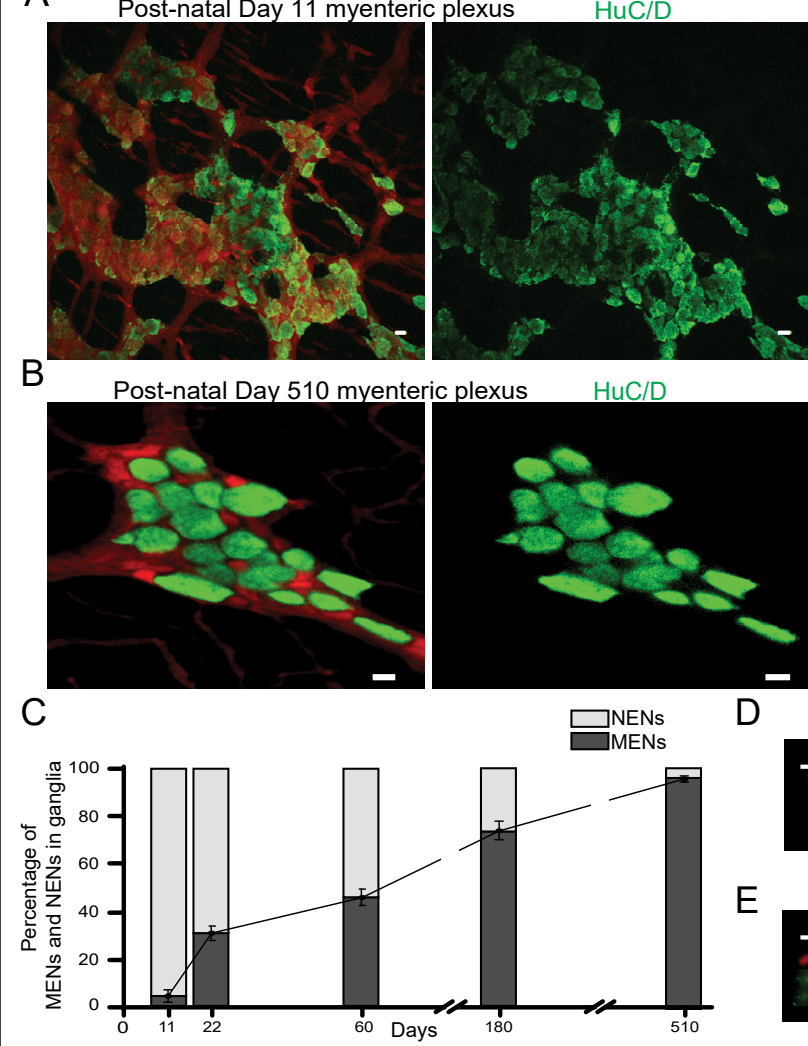

$\mathrm{F}$

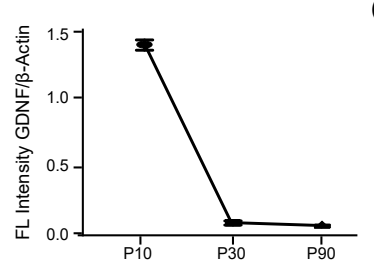

I GDNF suppl in P11
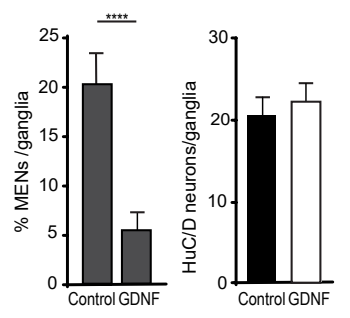

$\mathrm{G}$

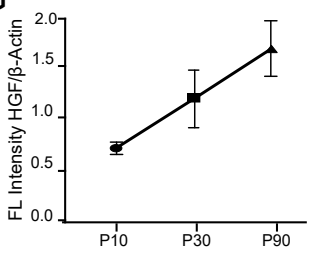

$J$

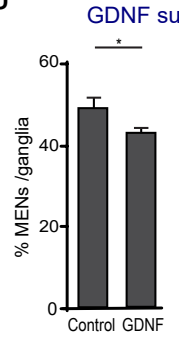

D

Wnt1-cre:Rosa26-tdTomato

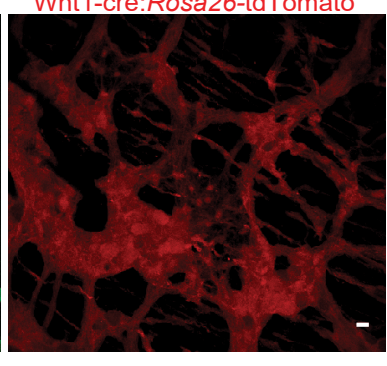

Wnt1-cre:Rosa26-tdTomato

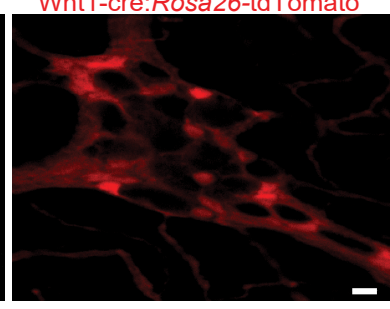

B-Actin GDNF

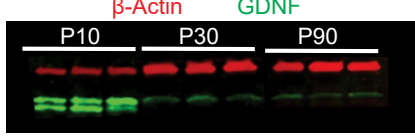

E

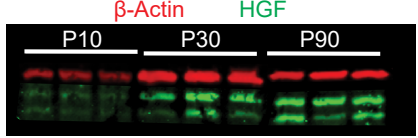

$\mathrm{H}$

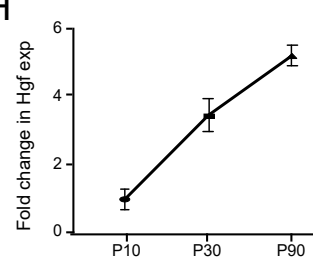

K

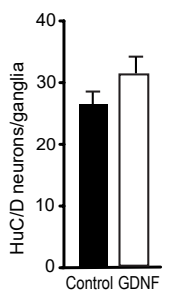

HGF suppl in P11

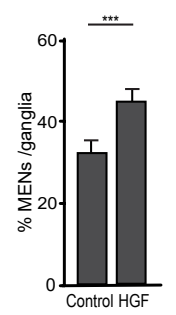

Fig 5: Age-dependent changes in proportions of the two neuronal lineages are governed by GDNF and HGF signaling.

(A, B) Immunostaining myenteric plexus tissue from juvenile and mature Wnt1-cre:Rosa26-tdTomato mice with antibodies against the pan-neuronal marker HuC/D (green). (A) Only a few neurons do not express tdTomato in $\mathrm{P} 11$ mice, indicating the ENS neuronal population at this stage consists largely of NENs. (B) All HuC/D+ neurons in P510 mice are tdTomato- MENs. Scale bar $=10 \mu \mathrm{m}$. (C) The proportion of the ENS contributed by neurons of the NEN lineage dwindles rapidly in maturing Wnt1-cre:Rosa26-tdTomato mice and continues to do so with further aging. This is associated with a corresponding increase in the proportion of ENS neurons derived from the MEN lineage. $(n=34$ ganglia from 3 mice for $\mathrm{P} 11 ; \mathrm{n}=45$ ganglia from 3 mice at $\mathrm{P} 22 ; \mathrm{n}=$ 37 ganglia from 3 mice at P60; $n=60$ ganglia from 6 mice at P180; and $n=67$ ganglia from 3 mice at P510). Data represent mean \pm S.E.M. Simple Linear Regression was used for statistical analyses. ( $F=$ 296.0, DFn, DFd = 1, 250; $p<0.0001$ ). (D) Western blot analyses of the expression of GDNF (green) and the house-keeping protein $\beta$-actin (red) in LMMP tissues from mice of ages P10, P30, and P90. ( $n=3$ mice per group; each sample is a biological replicate). Fluorescent intensities of the two bands of GDNF (that correspond to $\sim 25 \mathrm{kD}$ bands of protein marker) were quantified together. The lower band of GDNF is present only in the P10 tissues and disappears in P30 and P90 adult murine tissues. (E) Western blot analyses of the expression of HGF (green) and the house-keeping protein $\beta$-Actin (red) in LMMP tissues from mice of ages P10, P30, and P90. ( $n=3$ mice per group; each sample is a biological replicate). Fluorescent intensities of the two bands of HGF (that are between $50 \mathrm{kD}$ and $37 \mathrm{kD}$ bands of the protein marker) were quantified together. $(F)$ The normalized fluorescent intensity of GDNF protein to house-keeping protein $\beta$-Actin compared between the three age groups. GDNF presence was highest in P10 group. As compared with that in the P10 group, GDNF expression was significantly reduced in P30 and P90 groups. Data represent mean \pm S.E.M. Simple Linear Regression was used for statistical analyses. ( $F=6.821$, DFn, $D F d=1,7 ; p=0.0348)$. (G) The normalized fluorescent intensity of HGF protein to house-keeping protein $\beta$-Actin was compared between the three age groups. HGF expression significantly increased from P10 through P90. Data represent mean \pm S.E.M. Simple Linear Regression was

used for statistical analyses. ( $F=8.820$, DFn, DFd $=1,7 ; p=0.02$ ). $(H)$ Age-dependent increase in Hgf mRNA transcript expression (normalized to the house-keeping gene Hprt) in the myenteric plexi from P10, P30, and P90 old mice. Data represent mean \pm S.E.M. Simple Linear Regression was used for statistical analyses. ( $F=36.98$, DFn, DFd $=1,16 ; p<0.0001)$. (I) GDNF administration to Wnt1-cre:Rosa26-tdTomato mice from P10 to P20 age significantly reduced the proportion of HuC/D+tdTomato- MENs, as compared with saline-treated controls $(n=65$ ganglia from 3 mice for Controls; $n$

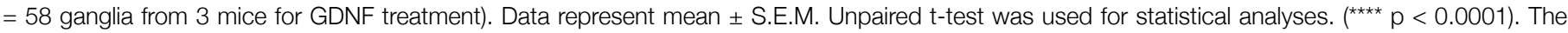
total numbers of HuC/D+ neurons in the myenteric ganglia did not change between the two treatment groups. (J) GDNF administration to Wnt1-cre:Rosa26-tdTomato mice from P60 to P70 age significantly reduced the proportion of MENs, as compared with saline-treated controls $(\mathrm{n}=57 \mathrm{ganglia}$ from 5 mice for Controls; $n=47$ ganglia from 4 mice for GDNF treatment). Data represent mean \pm S.E.M. Unpaired t-test was used for statistical analyses. ( $\mathrm{p}<0.05)$. The total numbers of HuC/D+ neurons in the myenteric ganglia did not change between the two treatment groups. (K) HGF administration to Wnt1-cre:Rosa26-tdTomato mice from P10 to P20 age significantly increased the proportion of MENs, as compared with saline-treated controls ( $\mathrm{n}=99$ ganglia from 5 mice for Controls; $n=104$ ganglia from 5 mice for HGF treatment). Data represent mean \pm S.E.M. Unpaired t-test was used for statistical analyses. $\left.{ }^{* \star *} \mathrm{p}<0.001\right)$. The total numbers of HuC/D+ neurons in the myenteric ganglia did not change between the two treatment groups.

et al., 2015). MENs were significantly larger than NENs in adult Wnt1-cre:Rosa26-tdTomato mice (Fig S4A), which is consistent with a putative role as IPANs which are known to be large diameter neurons (Mao et al., 2006). In addition, MENs-specific expression of another protein
Cadherin-3 (CDH-3; Fig 3H), which marks a sub-population of neurons in the spinal cord mechanosensory processing region (Abraira et al., 2017), supports a possible sensory function for some MENs. 


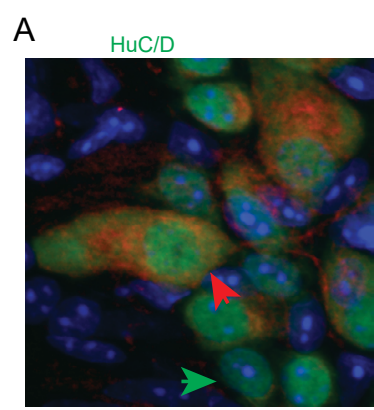

B

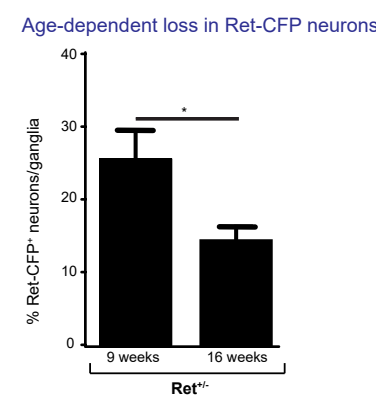

D

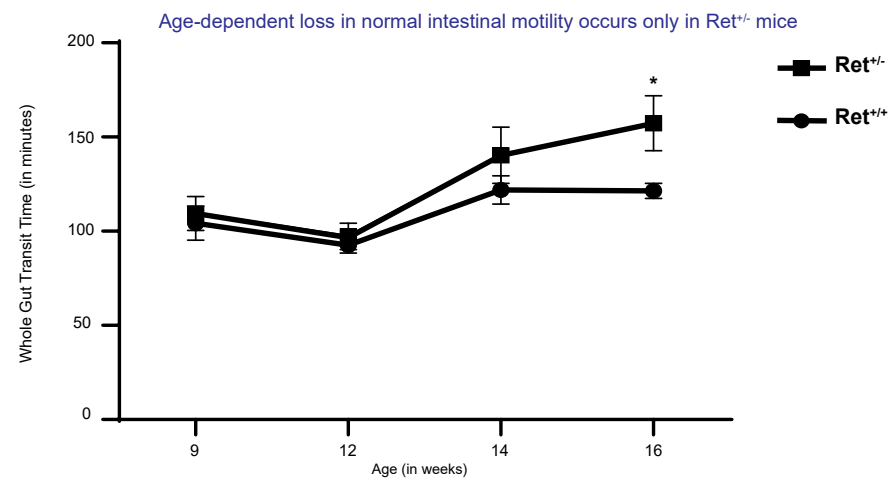

C
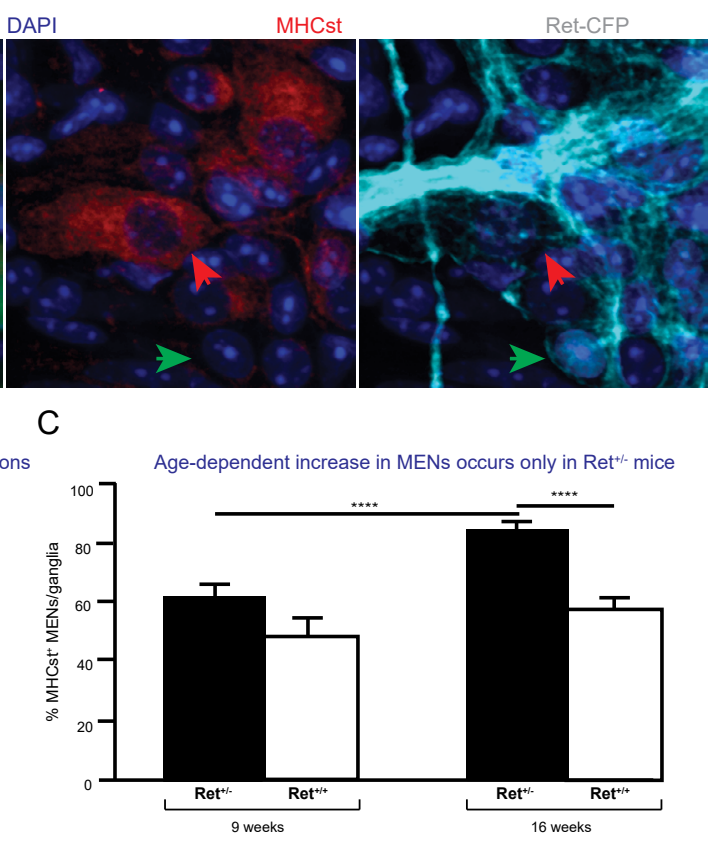

$t^{+/-}$ $t^{+/+}$
Single Cell RNA transcriptomics-based analyses further characterizes the novel MEN population

To further ascertain the separate nature of the MENs from NENs, we performed single cell RNA sequencing (scRNAseq) with the 10X Genomics Chromium V2.0 platform on all cells isolated from ileal LM-MP tissues from two 6-month-old adult male C57BL/6 wildtype mice. Such analyses have previously been performed on FACS-sorted Wnt1-cre:Rosa26-tdTomato ${ }^{+}$neural crest-derived cells from young adult mice (Zeisel et al., 2018). Here, we performed agnostic clustering of the scRNAseq data and found the presence of diverse cell-types in the LM-MP layer (Fig S4B, C). We identified the cells of the NEN lineage by expression of canonical neural crest markers Ret and Sox10 (Fig 4A, Fig S4B). We then identified the cluster containing MENs by its co-expression of the genes Calcb (CGRP), Met, and Cdh3 that we validated, as described above (Fig 3), to be either expressed predominantly or uniquely by MENs (Fig 4A, B, Fig S4B). With cells from both samples pooled together, we compared 1,713 NENs with 2,223 MENs.

We then queried the scRNAseq data for additional MENs-specific marker genes and found that the MEN cluster was enriched in the expression of genes Slpi, Aebp1, Clic3, Fmo2, Smo, Myl7, and Slc17a9, whose expression by adult enteric neurons has not been previously described (Fig 4B). In addition, we also found that the MEN cluster was enriched in previously characterized ENS markers, such as Ntf3 and II18 (De Giorgio et al., 2000; Jarret et al., 2020) (Fig 4B). With immunochemistry and confocal microscopy, we validated the MEN-specificity of these markers (Fig 4C, Fig S3B - K).

The proportion of mesoderm-derived neurons expands with age to become the dominant population in the aging ENS

Since the ENS at birth is known to be derived solely from cells of the NC-lineage (Espinosa-Medina et al., 2017; Hao and Young, 2009; Laranjeira et al., 2011; Young et al., 2000; Young and Newgreen, 2001b), we next studied the temporal kinetics of the arrival and expansion of the MEN lineage and found that the relative proportions of these two populations changed with maturity and aging. Using Wnt1-cre:Rosa26-tdTomato mouse line, we enumerated the $\mathrm{HuC} / \mathrm{D}^{+}$tdTomato ${ }^{-} \mathrm{MENs}$ in tissues from mice of different ages. At P11, MENs were found only in few isolated myenteric ganglia (Fig 5A) and together 


\section{BioRxiv Preprint}

represented only $\sim 5 \%$ of all myenteric neurons (Fig $5 \mathrm{C}$ ), suggesting that they originate in the ENS shortly before $\mathrm{P} 11$. The proportion of MENs rises sharply thereafter: by P22, they account for $\sim 30 \%$ of all myenteric neurons; and at $\mathrm{P} 60$ they represent $\sim 45 \%$ of all myenteric neurons (Fig 5C). By P180 (6-month-old; Fig 5C), this proportion reaches $75 \%$ and in the aged small intestine of a P510 (17-month-old), the ENS is populated almost exclusively by MENs (Fig 5B, C).

\section{GDNF and HGF signaling regulates the} populations of the neural crest-derived and the mesoderm-derived neurons, respectively

GDNF-RET signaling is responsible for proliferation and neurogenesis from NC-derived ENS precursor cells during development as well as for the survival of Retexpressing enteric neurons (Gianino et al., 2003; Natarajan et al., 2002; Rodrigues et al., 2011; Taraviras et al., 1999). Similarly, HGF signaling has been shown to be essential for the proliferation of mesoderm-derived cells (Amano et al., 2002). Since we know that the expression of the GDNF A
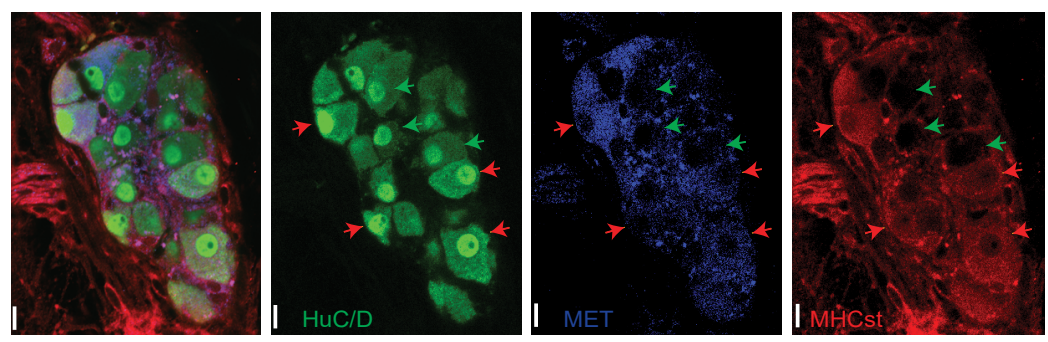

B
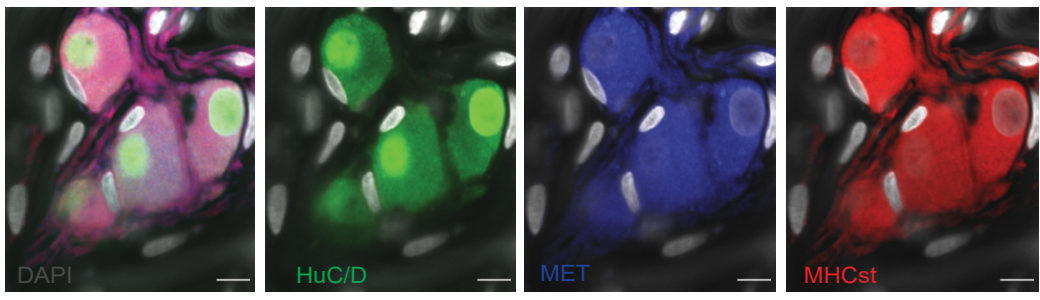

C
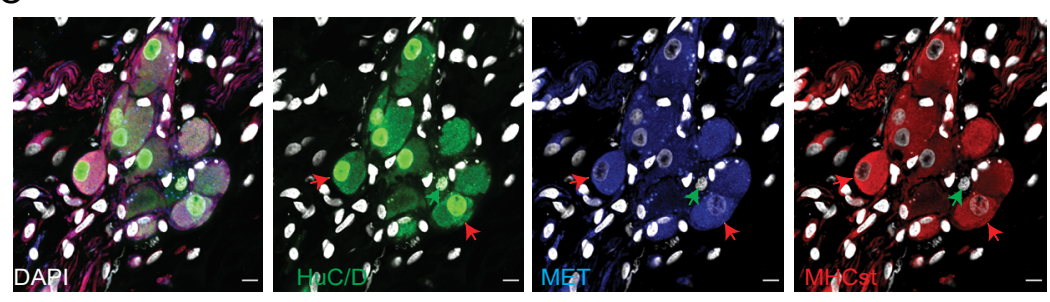

$\mathrm{D}$
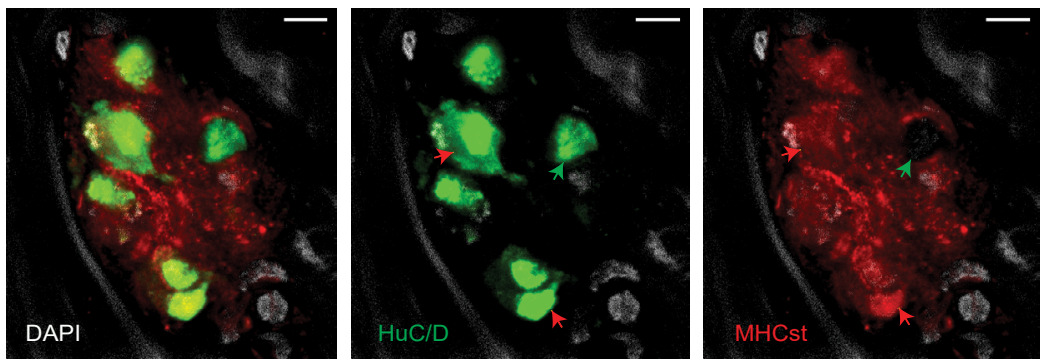

receptor RET and the HGF receptor MET occur exclusively on NENs and MENs, respectively, we next studied whether the changes in the two neuronal lineages during maturation are associated with changes in the levels of GDNF and HGF. We compared the expression of GDNF and HGF in the LM-MP tissue from terminal ileum of P10, P30, and P90 C57BL/6 wildtype mice and found that similar to a prior report (Wang et al., 2010), the expression of GDNF in the maturing ENS is markedly reduced between the P10 and the P30 developmental ages (Fig 5D, F). At the same time, the expression of HGF increases progressively from $\mathrm{P} 10$ to P90 (Fig 5E, G, H).

We next hypothesized that the reduced GDNF availability is responsible for the continual reduction in the proportion of NENs in the post-natal ENS. To test this, we administered GDNF or saline to cohorts of P10 Wnt1cre:Rosa26-tdTomato mice for 10 days (Gianino et al., 2003; Wang et al., 2010) and then counted HuC/D ${ }^{+} \mathrm{NENs}$ and MENs (based on the presence or absence of Wnt1-tdTomato expression, respectively). We found that GDNF treatment enhanced the proportions of NENs and correspondingly reduced the proportion of MENs in P20 mice to a level similar to that seen at the P10 age, while the proportion of MENs in the tissue from the salinetreated control mice remained at a level expected of its age (Fig 5I). Importantly, the expansion of the NEN lineage and associated contraction of the MEN lineage conserved the total neuronal numbers in the myenteric plexus (Fig $5 \mathrm{I}$ ), which is consistent with a previous report of the effect of GDNF supplementation on total neuronal numbers (Gianino et al., 2003). We then tested whether exogenous GDNF supplementation can also potentially reverse the age-associated loss of NENs in the adult ENS. To test this, we administered GDNF or saline to cohorts of male P60 Wnt1-cre:Rosa26-tdTomato mice. GDNF supplementation in adult mice significantly increased the proportions of NENs compared to the saline-treated control mice, while again maintaining the total neuronal numbers (Fig 5J).

Levels of HGF in the LM-MP tissue increase

Fig 7: Putative mesoderm-derived enteric neurons (MENs) are present in adult human myenteric ganglion.

(A), (B), and (C) The mesodermal specific markers, MET and MHSct are expressed by a subpopulation of human enteric neurons. Myenteric ganglia from normal duodenal tissue of 3 different adult human subjects express the pan-neuronal marker HuC/D (green), with distinct subpopulations positive and negative for MET (blue) and MHCst (red). MET and MHCst-expressing neurons (red arrows) in the human ENS are assumed to be mesoderm-derived enteric neurons (MENs) and those not expressing these MENs markers (green arrows) are presumably NENs. Nuclei in panels (B) and $(\mathrm{C})$ are labeled with DAPI (gray). Scale bar $=10 \mu \mathrm{m}$.

(D) Colonic human myenteric neurons expressing HuC/D (green) contain two populations of neurons that can be differentiated by their expression of MENs marker MHCst (red arrows). Nuclei are labeled with DAPI (gray). Scale bar $=10 \mu \mathrm{m}$ 
with age (Fig 5E, G, H) and we hypothesized that this is responsible for the continual expansion of MENs observed in the maturing ENS. We administered HGF and saline to cohorts of P10 Wnt1-cre:Rosa26-tdTomato mice every other day for 10 days in a manner similar to prior studies (Mizuno et al., 1998). Exogenous HGF significantly increased the proportions of MENs in P20 mice (Fig 5K) to levels that were similar to P60 mice in our previous observations (Fig 5C). At the same time, the proportion of MENs in the saline-treated control mice remained at its expected levels (Fig 5K). Also, similar to the GDNF experiment, providing exogenous HGF to the maturing ENS did not alter the total numbers of neurons in the small intestine (Fig $5 \mathrm{~K}$ ).

Having shown that exogenous augmentation of GDNFRET signaling increased the proportions of NENs in the adult gut, we then addressed the role of endogenous RET signaling in maintaining this population. Ret-null heterozygous mice have been previously used as a model to study the effect of reduced RET signaling in the adult ENS (Gianino et al., 2003). Mice carrying a RetCFP allele have a CFP reporter inserted at their Ret locus rendering it null (Uesaka et al., 2008). We used adult Ret ${ }^{+/ C F P}$ (or Ret ${ }^{+/-}$) mice that were 9 weeks $(\sim P 60)$ and 16 weeks ( P110) of age to study the effect of partial loss of Ret signaling in the adult ENS. We first confirmed that the NENs marker RetCFP, and MENs marker MHCst were expressed by different subpopulations of neurons (Fig 6A). Partial loss of Ret in the $\mathrm{Ret}^{+/-}$mice causes a significant loss in the numbers of Ret$\mathrm{CFP}^{+}$neurons with age (Fig 6B). At the same time, we found a significant increase in the proportion of $\mathrm{MHCst}^{+} \mathrm{MENs}$ with age in the Ret ${ }^{+/-}$mice (Fig 6C). By contrast, in the 9and 16-week control $\operatorname{Ret}^{+/+}$mice, we found no significant age-associated change in the proportions of MENs during this 7-week time-course (Fig 6C), thereby confirming that depletion of endogenous GDNF-RET signaling in the adult ENS accelerates the progressive loss of NENs with age.

\section{A shift in the balance between the two lineages in the adult ENS has functional consequences}

In humans with severe intestinal dysmotility, prior reports have found that there is a loss of expression of Gdnf, Ret, and its cognate receptor Gfra1 (Kim et al., 2019), while total numbers of enteric neurons are preserved (Bassotti et al., 2012). Since we observed that a loss of Ret expression in adults is associated with an accelerated loss of NENs, we hypothesized that this loss would be associated with progressive deterioration in intestinal function. Therefore, we studied the intestinal transit of a cohort of adult $\mathrm{Ret}^{+/-}$ mice and their littermate control $\operatorname{Ret}^{+/+}$mice over a timecourse of 7 weeks, starting from an age of 9 weeks till they were 16 weeks old. While the 9 week old adult $\operatorname{Ret}^{+/-}$ mice had similar whole gut transit times as their littermate control $\mathrm{Ret}^{+/+}$mice, the whole gut transit times between the two genotypes diverged with age; 16 week old $\mathrm{Ret}^{+/-}$ mice displayed significantly delayed intestinal transit, when compared to age-matched control $\mathrm{Ret}^{+/+}$mice (Fig 6D), consistent with previous reports of physiological deficits in intestinal contractility of Ret hypomorph mice at this age (Gianino et al., 2003).

\section{The adult human ENS also contains neurons that express mesodermal markers}

We performed immunostaining for $\mathrm{HuC/D}$, MHCst, and MET on small intestinal myenteric ganglia using surgically resected tissue from 3 adult patients with no known gut motility disorder (Fig 7A-C). We also performed immunohistochemistry using antibodies specific to HuC/D and MHCst on colonic LM-MP sample from a $4^{\text {th }}$ human donor who similarly had no known gut motility disorder (Fig 7D). Both $\mathrm{MHCst}^{+} \mathrm{MET}^{+} \mathrm{MENs}$ and $\mathrm{MHCst}^{-} \mathrm{MET}{ }^{-}$ NENs were observed to be present in the duodenal and colonic specimens, suggesting that the human ENS also contains neurons that express markers that can be used to identify the mesodermal lineage of enteric neurons in adult mice (Fig 7).

\section{DISCUSSION}

Current dogma states that the adult ENS is exclusively derived from neural crest precursors that populate the gut in early embryonic life (Young and Newgreen, 2001a). The results of this study indicate a much more dynamic system, one in which the fetal and early life "juvenile" ENS consisting of neural crest-derived enteric neurons (NENs) is incrementally replaced during maturation by mesodermderived enteric neurons (MENs) and that eventually the aging ENS consists almost exclusively of the neurons of the MEN lineage. This study also provides the first definitive evidence of a significant mesodermal contribution to any neural tissue. Previously, the mesoderm and especially the Lateral Plate Mesoderm (LPM) was known to give rise to diverse cell-types within the viscera, including several in the gut (Prummel et al., 2020), and our study shows that cells of this embryonic derivation also give rise to ENS neurons, that were previously thought to be solely derived from the neural crest. A previous report on a dual origin of the ENS described adult enteric neurons from Foxa2 ${ }^{+}$ or Sox $17^{+}$precursors (Brokhman et al., 2019) and inferred that these were endodermal in origin. However, Foxa2 and Sox17 are also expressed by mesoderm-derived cells (Bardot et al., 2017; Burtscher et al., 2012; Kawaguchi et al., 2007; Wilm et al., 2005). By contrast, using two NClineage-traced mouse lines (Wnt1-cre and Pax3-cre), two lineage-traced mouse lines marking mesodermal derivatives (Tek-cre, and Mesp1-cre), and robust adult mesoderm markers, we identify a population of Mesp1derived MHCst- and MET-expressing adult enteric neurons, which makes up the entire non-NC-derived population of myenteric neurons. These results provide evidence that the second source of adult enteric neurons is the mesoderm, and not the endoderm. Importantly, we were also able to confirm MHCst and MET expression in many enteric neurons in adult humans, suggesting that the mesoderm-derived ENS may be a feature common to mice and humans alike. MENs in both species can readily be 
identified by their expression of MHCst, CDH-3 and MET, thus providing a convenient tool to further discriminate and study these neurons.

In our single cell transcriptomic analysis, we used the markers Calcb, Met, and Cdh3 that we had validated to identify and annotate the MEN cell cluster. When compared with the Ret and Sox10-expressing NEN cluster, an additional set of marker genes were found to be expressed widely or selectively within MENs. Some of these, such as Slpi, Aebp1, Clic3, and Fmo2 have not been previously described in enteric neurons, while others (Ntf3, II18, and Cftr) are known to be expressed (De Giorgio et al., 2000; Jarret et al., 2020; Xue et al., 2016). The differential expression of genes between the two neuronal populations (MENs versus NENs) suggests putative specialized functional roles of these subpopulations when they co-exist in adults. Prior scRNAseq analysis of postnatal ENS did not detect and characterize the true identity of the MEN lineage. This is because previous studies were performed either exclusively on neural crest-derived ENS cells (Zeisel et al., 2018), or when done in a more agnostic manner, only applied known canonical neural markers to identify the ENS population (Drokhlyansky et al., 2019; Morarach et al., 2020). In either case, the discovery of the distinct identity and derivation of MENs would not have been possible.

Since our scRNAseq data highlighted the lineagespecific nature of the expression of Ret and Met, we studied whether these genes regulated the origin, expansion, and maintenance of the neuronal populations that express them. While it is known that Gdnf expression in the mature gut is significantly downregulated (Avetisyan et al., 2015; Gianino et al., 2003; Wang et al., 2010), the functional consequences of this loss have been unclear (Gianino et al., 2003). We found that reduced GDNF-RET signaling drives the age-dependent loss of NENs as this loss can be retarded or reversed by GDNF supplementation and accelerated by further reduction of Ret expression. Our results identify a novel role for GDNF in maintaining the canonical NEN population, keeping the MEN population in check, while preserving overall enteric neuronal numbers. In the last few years, studies have focused on identifying juvenile protective factors (JPFs), the loss of which correlates with or drives maturation- and agingassociated phenotypes (Papaconstantinou et al., 2015). In this context, GDNF may therefore qualify as a JPF as its presence maintains the dominance of NENs in ENS, corresponding to a juvenile phenotype.

The observed mutually exclusive expression of Ret and Met by the two lineages of enteric neurons in the mature adult ENS is consistent with an earlier study that reported that a deletion of Met in the Wnt1-cre expressing $\mathrm{NC}$-lineage did not alter the abundance of $\mathrm{MET}^{+}$neurons in the adult ENS (Avetisyan et al., 2015). In this study, we also show that MENs are dependent on HGF-MET signaling in a manner analogous to the requirement of GDNFRET signaling by NENs. Further, Ret haploinsufficiency- mediated loss of the NEN lineage causes a proportional increase in the MEN lineage. This suggests the presence of yet unknown quorum sensing signaling mechanisms between the two lineages that reciprocally regulate their populations to maintain the structure of the post-natal ENS. This also implies the presence of a yet unknown precursor cell responsible for the contraction and expansion of the MEN population in a manner analogous to what we have previously described for NENs (Kulkarni 2017).

The validation of mesodermal markers (MHCst and MET) in a subset of adult human ENS neurons suggests that our findings may be of clinical importance. The loss of NENs and the corresponding dominance of MENs begins in early adulthood and maybe viewed as part of the normal maturation of the ENS. However, because of its progressive nature, it may have pathological implications for the elderly gut. Many gastrointestinal motility functions are compromised with advancing age, resulting in clinically significant disorders (Saffrey, 2014). A progressive imbalance in these two populations in favor of MENs with age may therefore have functional consequences. Although the exact mechanisms will need to be worked out, our results indicate that a MENs-predominant ENS is associated with significant differences in gut motility. Understanding the forces that regulate parity between these two different sources of neurogenesis therefore holds the promise of arresting or reverting progressive loss of gut motility with increasing age. Our results may also have implications for the pathogenesis of disordered motility unrelated to aging, as downregulation of Gdnf and Ret expression has been associated with diverticular disease and obstructed defecation in adult patients (Barrenschee et al., 2017; Cossais et al., 2019; Kim et al., 2019). GWAS analyses further showed that Gdnf gene was in the eQTL associated with increased incidence of diverticular disease (Schafmayer et al., 2019).

In conclusion, the ENS of the juvenile and mature mammalian gut is dominated by two different classes of neurons that have distinct ontogenies and trophic factors, associated with functional differences. This may represent an adaptation to differences in nutrient handling, microbiota or other physiological factors as the organism transitions through various stages of life. Further research in the regulation of the parity between these two nervous systems in humans during the course of the lifetime will be important to advance our understanding of the adult ENS and the treatment of age-related and other pathological disorders of gut motility.

\section{ACKNOWLEDGEMENTS}

We would like to thank Dr. Chulan Kwon (JHU) for his kind gift of the Mesp1-cre mice, Dr. Jeremy Nathans (JHU) for his kind gift of the Tek-EGFP, Tek-cre and Hprt-tdTomato lineage-traced mice and his support, and Dr. Vanda Lennon (Mayo Clinic) for her kind gift of the ANNA1 anti$\mathrm{Hu}$ antisera. We thank Dr. Xinzhong Dong (JHU) and Dr. Mark Donowitz (JHU) for their support. The microscopy was performed on the Ross Imaging Core at the Hopkins Conte 
Digestive Disease Center at the Johns Hopkins University (P30DK089502) and we thank Dr. George McNamarra for his help with confocal training and imaging. The $10 X$ Genomics Chromium processing for scRNAseq was performed the GRCF Core and the sequencing was performed at the CIDR core at the Johns Hopkins University. S.K. was funded through a grant from the Ludwig Foundation and a pilot award from the Hopkins Digestive Diseases Basic \& Translational Research Core Center grant P30DK089502. L.A.G was funded in part by a Johns Hopkins Catalyst Award. P.J.P. was funded through the Hopkins Conte Digestive Disease Center at the Johns Hopkins University (P30DK089502), NIDDK (R01DK080920), the Maryland Stem Cell Research Foundation (MSCRF130005), and a grant from the AMOS family.

\section{AUTHOR CONTRIBUTIONS}

S.K., and P.J.P. conceived the study; S.K., M.A., M.L, L.A.G. and P.J.P. designed the research studies; S.K. L.B, and M.S. conducted the experiments; S.K., Z.H., M.K., J.L., L.B., S.B., E.V., J.S., S.N., M.S., G.L., and L.A.G. acquired and analyzed the data; S.K., and P.J.P drafted the manuscript.

\section{METHODS}

\section{Animals}

Experimental protocols were approved by The Johns Hopkins University's Animal Care and Use Committee in accordance with the guidelines provided by the National Institutes of Health. Presence of vaginal plug was ascertained as 0.5 days post-fertilization and this metric was used to calculate age of mice. Only male mice were used for the studies detailed in this report. The Wnt1cre:Rosa26-tdTomato lineage-traced line was generated as detailed before by breeding the B6.Cg-Tg(Wnt1-cre) with the Ai14 transgenic mouse line (Jax \#: 007914) containing the Rosa26- tdTomato transgene(Becker et al., 2012; Becker et al., 2013; Kulkarni et al., 2017). Pax3cre:Rosa26-tdTomato lineage-traced line was generated by breeding the Ai9 transgenic mouse line (Jax \#: 007909) with the Pax3-cre transgenic mouse (Jax \#: 005549). The Wnt1-cre:Hprt-tdTomato mouse was generated by breeding our aforementioned Wnt1-cre transgenic mouse line with the Hprt-tdTomato transgenic mouse line (Jax \#: 021428, kind gift from Prof. Jeremy Nathans). Mesp1-cre:Rosa26tdTomato mice were generated by breeding the Mesp1-cre transgenic mice (Shenje et al., 2014) (gift from Dr. Chulan Kwon, JHU) with the Ai14 transgenic mice. Ret ${ }^{+/ C F P}$ mice (MGI:3777556) were inter-bred to get a colony of adult Ret ${ }^{+++}$ and Ret $^{+/ C F P}$ mice. Ret ${ }^{\text {CFP/CFP }}$ mice died at or before term. Tek-cre:Hprt-tdTomato mice were generated by breeding Tek-cre transgenic mice (also known as Tie2-cre; Jax \#: 004128) with the Hprt-tdTomato transgenic mouse line.

\section{Human Tissues}

Human tissues were obtained under IRB protocol
IRB00181108 that was approved by Institutional Review Board at the Johns Hopkins University. Pathological normal specimens of human duodenum and colon were obtained post-resection. Tissues were obtained from adult donors and were de-identified such that the exact age, gender, and ethnicity of the donors was unknown.

\section{Tissue preparation}

Mice were anesthetized with isoflurane and sacrificed by cervical dislocation. A laparotomy was performed, and the ileum was removed and lavaged with PBS containing penicillin-streptomycin (PS; Invitrogen), then cut into 1-cmlong segments and placed over a sterile plastic rod. A superficial longitudinal incision was made along the serosal surface and the LM-MP was peeled off from the underlying tissue using a wet sterile cotton swab (Kulkarni et al., 2017) and placed in Opti-MEM medium (Invitrogen) containing Pen-Strep (Invitrogen). The tissue was then laid flat and fixed with freshly prepared ice cold $4 \%$ paraformaldehyde (PFA) solution for 45 minutes in the dark to preserve fluorescence intensity and prevent photo-bleaching. After the fixation, the tissue was removed and stored in ice cold sterile PBS with Pen-Strep for immunofluorescence staining and subsequent microscopy.

For human tissues, duodenal tissue from adult human patients ( $n=3$ patients), who did not have any prior history of chronic intestinal dysmotility, that was removed by Whipple procedure was obtained. A colonic sample from a pathologically normal colonic resection from an adult donor suffering from colon carcinoma who similarly did not have prior history of chronic intestinal dysmotility was also obtained. The resected tissue was placed in ice cold Opti-MEM medium (Invitrogen) containing PenStrep (Invitrogen). The mucosal and sub-mucosal tissue was dissected out in the medium under light microscope and the muscularis layer containing myenteric plexus tissue was obtained. The tissue was laid out between two glass slides and fixed overnight in ice cold 4\% PFA after which it was removed and stored in ice cold sterile PBS with Pen-Strep for immunofluorescence staining, optical clarification and subsequent microscopy.

\section{Immunohistochemistry}

For murine tissue: The fixed LM-MP tissue was washed twice in ice-cold PBS in the dark at $16^{\circ} \mathrm{C}$. The tissue was then incubated in blocking-permeabilizing buffer (BPB; $5 \%$ normal goat serum with $0.3 \%$ Triton-X) for 1 hour. While staining for antibodies that were mouse monoclonal, $5 \%$ normal mouse serum was added to the BPB. The tissue was then removed from the BPB and was incubated with the appropriate primary antibody at the listed concentration (Table S1) for $48 \mathrm{~h}$ at $16^{\circ} \mathrm{C}$ in the dark with shaking at $55 \mathrm{rpm}$. Following incubation with primary antibody, the tissue was washed three times (15-min wash each) in PBS at room temperature in the dark. The tissue was then incubated in the appropriate secondary antibody at room temperature for 1 hour while on a rotary shaker (65 rpm). The tissue was again washed three times in PBS at room temperature, counterstained with DAPI to stain 
the nuclei, overlaid with Prolong Antifade Gold mounting medium, cover-slipped, and imaged.

Colchicine treatment: For CGRP immunostaining, mice were injected with Colchicine at a concentration of 5 $\mathrm{mg} / \mathrm{Kg}$ body weight 16 hours (overnight) before they were sacrificed. The mice were housed singly during this time and adequate gel packs were provided. Food and water were provided ad libitum. On the following day, the mice were sacrificed, and their LM-MP tissues were harvested as detailed above.

For human tissue: The fixed muscularis layer containing myenteric plexus tissue was removed from ice cold PBS and incubated in blocking-permeabilizing buffer (BPB; $5 \%$ normal goat serum, $5 \%$ normal mouse serum with $0.3 \%$ Triton-X) for 4 hours. The tissue was then removed from the BPB and was incubated with the appropriate primary antibody at the listed concentration (Table S1) for 5 days at $16^{\circ} \mathrm{C}$ in the dark with shaking at 55 rpm. Following incubation with primary antibody, the tissue was washed five times (15-min wash each) in PBS at room temperature in the dark. The tissue was then incubated in the appropriate secondary antibody at $16^{\circ} \mathrm{C}$ in the dark with shaking at $55 \mathrm{rpm}$ for 2 days. The tissue was again washed in dark for five times in PBS that contained DAPI at room temperature. After the final wash, the tissue was suspended in tissue clarification buffer CUBIC (Susaki et al., 2014) for 1 hour at $4^{\circ} \mathrm{C}$ in the dark after which it was overlaid with Prolong Antifade Gold mounting medium, cover-slipped, and imaged. Briefly, the CUBIC optical clarification buffer was made by mixing $2.5 \mathrm{~g}$ of urea $(25 \%$ by wt), $2.5 \mathrm{~g}$ of $\mathrm{N}, \mathrm{N}, \mathrm{N}^{\prime}, \mathrm{N}$-'tetrakis (2-hydroxy-propyl) ethylenediamine ( $25 \%$ by wt), $1.5 \mathrm{~g}$ of Triton X-100 ( $15 \%$ by wt) in $35 \mathrm{ml}$ of Distilled Water. The solution was shaken till the ingredients were dissolved and yielded a clear viscous solution.

\section{Microscopy}

Imaging was done by using the oil immersion $63 \mathrm{X}$ objective on the Leica SP8 confocal microscope and by using the oil immersion 40X objective on the Olympus Fluoview 3000rs confocal microscope with resonance scanning mode. For thick tissues, such as human tissues, the Galvano mode of the Olympus Fluoview 3000rs microscope that enabled higher resolution imaging and averaging was used. Images obtained were then analyzed using Fiji (https://fiji.sc/).

\section{Enumeration of neurons}

Identification of myenteric ganglia was performed according to our pre-determined method published earlier (Kulkarni et al., 2017). Briefly, contiguous clusters of neurons were defined as a ganglia and the total numbers of neurons within these clusters were enumerated as numbers of myenteric neurons per ganglion. As a rule, clusters of 3 neurons or more were deemed to consist a ganglion and our enumeration strategy did not count extra-ganglionic neurons. We imaged atleast 10 ganglia per tissue for our enumeration and each group studied had $n \geq 3$ mice. Identification and enumeration of neurons and detection of co-localization was performed manually by trained laboratory personnel.

\section{Protein isolation and detection}

After the LM-MP tissue was isolated, it was weighed and placed in a sterile $1.5 \mathrm{ml}$ microfuge tube. 1X RIPA buffer (Cell Signaling Technology) with Halt Protease Inhibitor Cocktail (Thermo Scientific) at 5X concentration, Phosphatase Inhibitor Cocktails II and III (Sigma-Aldrich) at $2 X$ concentrations were added to the tissue lysate buffer. Tissue was disrupted using $1.0 \mathrm{~mm}$ silica beads in Bullet Blender 24 (Next Advance) for 5 mins at highest setting. The lysate was incubated at $4^{\circ} \mathrm{C}$ with shaking for 30 mins, centrifuged at $14,000 \mathrm{rpm}$ for 20 mins and the supernatant was taken and stored in $-80^{\circ} \mathrm{C}$ in aliquots. Protein concentration was estimated using Bradford assay solution (Biorad) following the manufacturer's protocol. For immunoblotting, $40 \mu \mathrm{g}$ of protein was loaded per well of $4 \%$ $20 \%$ gradient denaturing gel (Biorad). Protein marker used was Precision Plus Dual Color standards (Biorad). After fractionating the proteins, they were blotted onto ImmunBlot PVDF membrane (Biorad) overnight at $4^{\circ} \mathrm{C}$ at $17 \mathrm{~V}$ for $12-16$ hours. After blotting, membrane was blocked with Odyssey TBS blocking buffer (Li-Cor) for 1 hour at room temperature with shaking. Incubation with primary antibodies were carried out at $4^{\circ} \mathrm{C}$ with shaking for 24 hours. Following binding, the blot was washed 4 times with TBS-T (Tris Buffered Saline with $0.5 \%$ Tween) for 15 mins each with shaking at room temperature. Secondary antibody incubation was carried out in dark at room temperature for 1.5 hours with shaking. The blot was then washed 4 times for 15 mins each and imaged on Odyssey CLx system (Li-Cor). Antibodies used are detailed in the Table S1.

\section{RNA isolation and quantitative detection of specific transcripts}

The isolated tissue was stored in RNALater Solution (Ambion). RNA was isolated using RNeasy Mini Kit (Qiagen) following manufacturer's protocol. RNA quantification was carried out using Epoch Microplate Spectrophotometer (BioTek). cDNA synthesis was carried by SuperScript IV VILO Master Mix (Invitrogen). Quantitative Real-time PCR was carried out using Taqman Gene Expression Master Mix (Applied Biosystems) and Roto-Gene Q (Qiagen). The probes used are listed in Table S1.

\section{Single Cell RNA sequencing and analyses}

Single cell preparation from adult murine ileal tissues: Ileal tissues from two 6-month old adult male littermate C57/ BL6 wildtype mice were isolated by peeling as previously described. The tissues were then dissociated in Digestion Buffer containing $1 \mathrm{mg} / \mathrm{ml}$ Liberase (Sigma-Aldrich) in OptiMEM. Tissues from mouse 1 were dissociated in the Digestion buffer containing Liberase $\mathrm{TH}$ and tissues from mouse 2 were dissociated in the Digestion buffer containing Liberase TL. Dissociation was performed at $37^{\circ} \mathrm{C}$ for 30 minutes on a rotary shaker, after which the cells were centrifuged at $200 \mathrm{~g}$ for 7 minutes, and the pellet was resuspended in ice cold sterile PBS. The cell suspension 
was passed through a $40 \mu \mathrm{m}$ cell sieve and the resulting filtered cell suspension was again centrifuged at $200 \mathrm{~g}$ for 7 minutes. This process of cell centrifugation and filtration was repeated two more times, after which the cells were resuspended in $1 \mathrm{ml}$ ice cold sterile PBS. The repeated steps of serial cell washes and filtration removed clumps and debris and the viability of the resulting cell suspension was estimated to be $>90 \%$ using Trypan Blue dye test. The cells were then processed through 10X Genomics Chromium V2.0 system according to the manufacturer's suggested workflow. The processing was done at the GRCF Core Facility at the Johns Hopkins University. The pooled libraries were sequenced on an Illumina HiSeq 2500 to an average depth of $3.125 \times 10^{8}$ reads per sample library. The sequencing was performed at the CIDR core facility at the Johns Hopkins University.

Pre-processing of FASTQs to Expression Matrices: FASTQ sequence files were processed following a Kallisto Bustools workflow compatible with downstream RNA velocity calculations [kallisto $==0.46 .1$, bustools $==0.39 .3$ ] (Bray et al., 2016). References required for pseudo-alignment of transcripts were obtained using the get_velocity_files() functionality of BUSpaRSE (https://github.com/BUStools/ BUSpaRse), with " $L=98$ " for $10 X$ Genomics $v 2.0$ sequencing chemistry. Reads were pseudo-aligned to an index built from Ensembl 97 transcriptome annotation (Gencode vM22; GRCm38). Across two samples processed, a total of $578,529,125$ reads were successfully pseudo-aligned. Barcodes within a Hamming distance of one to known 10X Genomics v2.0 barcodes were corrected. Reads were classified as "spliced" or "unspliced" by their complement to the target list of intronic sequences and exonic sequences, respectively, and subsequently quantified separately into expression count matrices. Spliced counts are used for all analyses.

\section{Single cell gene expression analysis}

scRNA-seq count matrices were analyzed using Monocle3. 11,123 high-quality cells were identified as meeting a $200 \mathrm{UMI}$ minimum threshold with a mitochondrial read ratio of less than $20 \%$; droplets that did not meet these criteria were excluded from the analysis. Mitochondrial counts were determined as the sum of reads mapping to 37 genes annotated to the mitochondrial genome. All genes with non-zero expression were included for downstream analysis. Raw counts were first scaled by a cell-size scaling factor and subsequently log10 transformed with a pseudocount of 1 . Normalized values are used in place of raw counts unless otherwise noted.

Prior to UMAP dimensionality reduction, batch effects between the two biological replicates were assessed and corrected via the mutual nearest neighbors (MNN) algorithm as implemented by Batchelor in Monocle3 (50 principal components, with default $\mathrm{k}=20$ ) (Haghverdi et al., 2018). 15 clusters of cells in the UMAP embedding were identified by Leiden community detection (resolution $=1 e-5$, number of iterations $=5$ ). 30 marker genes for each cluster were identified based on greatest pseudo $\mathrm{R}^{2}$ values and used for supervised annotation of cell types by searching UniProt, Allen Cell Atlas and through literature search with Pubmed.

NEN clusters were identified by expression of NC markers Ret and Sox10. MEN cluster was identified by its expression of CGRP-coding Calcb, Met, and Cdh3. The pan-MENs protein marker MHCst was identified by labeling with an antibody S46 which labels all members of the MHCst family (Stockdale and Miller, 1987). Since the antibody does not identify a single gene product, MHCst immunostaining could not be used to identify a specific gene marker for use in the annotation of the MEN cluster. For further analysis into the MENs population, the full LMMP dataset was subset to include only the 2,223 cells annotated as such. These cells were re-processed as above, but with a reduced PCA dimensionality of $k=20$ as input for the UMAP embedding. 5 clusters of cells in the UMAP embedding were identified by Leiden community detection $(k=10$, resolution $=5 e-4)$. The two samples showed a similar distribution of cells across the various cell clusters (Fig S3A). The MEN cluster had a significantly higher mean UMI count/cell compared to the NEN cluster (Mean UMI \pm S.E.M. of UMI/cell: MENs: $4045.6 \pm 113.4$; NENs: $1247.9 \pm 38.9$; $\mathrm{t}=23.34, \mathrm{df}=2727.8, \mathrm{p}<2.2 \mathrm{e}-16$; Welch two sample t-test; Fig S4D). Total UMI per cell is correlated directly with cell size (Padovan-Merhar et al., 2015). A significantly higher UMI/cell in MENs compared to NENs suggests that MENs would have significantly larger cell size compared to NENs, which we have confirmed in Fig S4A.

\section{Whole gut transit time analyses}

Whole-gut transit time for every mouse was analyzed by the method using the carmine red protocol (Kulkarni et al., 2017). Mice were placed in individual cages and deprived of food for 1 hour before receiving $0.3 \mathrm{~mL} 6 \%$ (wt/vol) carmine solution in $0.5 \%$ methylcellulose by oral gavage into the animal's stomach. The time taken for each mouse to produce a red fecal pellet after the administration of carmine dye was recorded in minutes. The mean difference in whole gut transit time (in minutes) between both the $\operatorname{Ret}^{+/+}$and $\mathrm{Ret}^{+/-}$mice were analyzed statistically.

\section{In vivo Injections}

GDNF injection: Similar to prior report that gave subcutaneous injections of GDNF to post-natal mice (Wang et al., 2010), we took 6 littermate 10 day old (P10) male Wnt1-cre:Rosa26-tdTomato mice and divided into two subgroups, GDNF and Control. Each mouse in the GDNF group was injected sub-cutaneously with $50 \mu \mathrm{l}$ of $2 \mathrm{mg} / \mathrm{ml}$ of GDNF (Peprotech Catalogue \#: 450-44) every other day, while the Control group was injected with $50 \mu \mathrm{l}$ of sterile saline. The mice were given 5 doses and then sacrificed on P20, after which their LM-MP tissues were isolated as detailed above. The tissues were then immunostained with antibodies against HuC/D and imaged. In a separate experiment, adult (P60) mice were also injected subcutaneously with GDNF (100 $\mu \mathrm{l}$ of $100 \mu \mathrm{g} / \mathrm{ml}$ of GDNF). The mice were given 5 doses over a course of 10 days and then sacrificed on P70, after which their LM-MP 
tissues were isolated as detailed above. The tissues were then immunostained with antibodies against HuC/D and imaged.

HGF injection: Similar to prior report that gave subcutaneous injections of HGF to post-natal mice, we took 6 littermate 10 day old (P10) male Wnt1-cre:Rosa26tdTomato mice and divided into two subgroups, HGF and Control. Each mouse in the HGF group was injected subcutaneously with $100 \mu \mathrm{l}$ of $2 \mathrm{mg} / \mathrm{ml}$ of HGF (Peprotech Catalogue \#:315-23) every other day, while the saline group was injected with $100 \mu \mathrm{l}$ of sterile saline. The mice were given 5 doses and then sacrificed on P20, after which their LM-MP tissues were isolated as detailed above. The tissues were then immunostained with antibodies against HuC/D and imaged.

\section{Statistics}

Data was analyzed using Graphpad Prism 8.3.1 and R using Unpaired Students t-test, Simple Linear Regression, and Ordinary One-Way ANOVA.

\section{Data Availability}

All raw data are provided in Supplementary Table 2. We imaged atleast 10 ganglia per tissue for our enumeration and each group studied had $n \geq 3$ mice. Raw single cell RNA sequencing data is archived on the NCBI GEO server and can be accessed under the accession number GSE156146.

\section{REFERENCES}

Abraira, V.E., Kuehn, E.D., Chirila, A.M., Springel, M.W., Toliver, A.A., Zimmerman, A.L., Orefice, L.L., Boyle, K.A., Bai, L., Song, B.J., et al. (2017). The Cellular and Synaptic Architecture of the Mechanosensory Dorsal Horn. Cell 168, 295-310 e219.

Amano, O., Yamane, A., Shimada, M., Koshimizu, U., Nakamura, T., and Iseki, S. (2002). Hepatocyte growth factor is essential for migration of myogenic cells and promotes their proliferation during the early periods of tongue morphogenesis in mouse embryos. Dev Dyn 223, 169-179

Anderson, R.B., Newgreen, D.F., and Young, H.M. (2006). Neural crest and the development of the enteric nervous system. Adv Exp Med Biol 589, 181-196.

Antal, M., Beneduce, B.M., and Regehr, W.G. (2014). The substantia nigra conveys target-dependent excitatory and inhibitory outputs from the basal ganglia to the thalamus. J Neurosci 34, 8032-8042.

Avetisyan, M., Wang, H., Schill, E.M., Bery, S., Grider, J.R., Hassell, J.A., Stappenbeck, T., and Heuckeroth, R.O. (2015). Hepatocyte Growth Factor and MET Support Mouse Enteric Nervous System Development, the Peristaltic Response, and Intestinal Epithelial Proliferation in Response to Injury. J Neurosci 35, 11543-11558.

Bardot, E., Calderon, D., Santoriello, F., Han, S., Cheung, K., Jadhav, B., Burtscher, I., Artap, S., Jain, R., Epstein, J., et al. (2017). Foxa2 identifies a cardiac progenitor population with ventricular differentiation potential. Nat Commun 8, 14428.

Barrenschee, M., Wedel, T., Lange, C., Hohmeier, I., Cossais, F., Ebsen, M., Vogel, I., and Bottner, M. (2017). No neuronal loss, but alterations of the GDNF system in asymptomatic diverticulosis. PLoS One 12, e0171416.

Bassotti, G., Villanacci, V., Bellomi, A., Fante, R., Cadei, M., Vicenzi, L., Tonelli, F., Nesi, G., and Asteria, C.R. (2012). An assessment of enteric nervous system and estroprogestinic receptors in obstructed defecation associated with rectal intussusception. Neurogastro- enterol Motil 24, e155-161.

Becker, L., Kulkarni, S., Tiwari, G., Micci, M.-A., and Pasricha, P.J. (2012). Divergent fate and origin of neurosphere-like bodies from different layers of the gut. American Journal of Physiology-Gastrointestinal and Liver Physiology 302, G958-G965.

Becker, L., Kulkarni, S., Tiwari, G., Micci, M.-A., Peterson, J., and Pasricha, P.J. (2011). Spatial and Temporal Differences Between Enteric Neural Progenitors From Small Intestine of Adult Mice. Gastroenterology 140 , S-522.

Becker, L., Peterson, J., Kulkarni, S., and Pasricha, P.J. (2013). Ex vivo neurogenesis within enteric ganglia occurs in a PTEN dependent manner. PLoS One 8, e59452.

Bergner, A.J., Stamp, L.A., Gonsalvez, D.G., Allison, M.B., Olson, D.P., Myers, M.G., Jr., Anderson, C.R., and Young, H.M. (2014). Birthdating of myenteric neuron subtypes in the small intestine of the mouse. $J$ Comp Neurol 522, 514-527.

Bray, N.L., Pimentel, H., Melsted, P., and Pachter, L. (2016). Near-optimal probabilistic RNA-seq quantification. Nat Biotechnol 34, 525-527.

Brokhman, I., Xu, J., Coles, B.L.K., Razavi, R., Engert, S., Lickert, H., Babona-Pilipos, R., Morshead, C.M., Sibley, E., Chen, C., et al. (2019). Dual embryonic origin of the mammalian enteric nervous system. Dev Biol 445, 256-270.

Burns, A.J. (2005). Migration of neural crest-derived enteric nervous system precursor cells to and within the gastrointestinal tract. Int $J$ Dev Biol 49, 143-150.

Burtscher, I., Barkey, W., Schwarzfischer, M., Theis, F.J., and Lickert, H. (2012). The Sox17-mCherry fusion mouse line allows visualization of endoderm and vascular endothelial development. genesis 50, 496505.

Chan, S.S., Shi, X., Toyama, A., Arpke, R.W., Dandapat, A., lacovino, M., Kang, J., Le, G., Hagen, H.R., Garry, D.J., et al. (2013). Mesp1 patterns mesoderm into cardiac, hematopoietic, or skeletal myogenic progenitors in a context-dependent manner. Cell Stem Cell 12, $587-$ 601.

Cossais, F., Lange, C., Barrenschee, M., Möding, M., Ebsen, M., Vogel, I., Böttner, M., and Wedel, T. (2019). Altered enteric expression of the homeobox transcription factor Phox $2 \mathrm{~b}$ in patients with diverticular disease. United European Gastroenterology Journal 7, 349-357.

De Giorgio, R., Arakawa, J., Wetmore, C.J., and Sternini, C. (2000). Neurotrophin-3 and neurotrophin receptor immunoreactivity in peptidergic enteric neurons. Peptides 21, 1421-1426.

Debbache, J., Parfejevs, V., and Sommer, L. (2018). Cre-driver lines used for genetic fate mapping of neural crest cells in the mouse: An overview. Genesis 56, e23105.

Devine, W.P., Wythe, J.D., George, M., Koshiba-Takeuchi, K., and Bruneau, B.G. (2014). Early patterning and specification of cardiac progenitors in gastrulating mesoderm. Elife 3.

Drokhlyansky, E., Smillie, C.S., Van Wittenberghe, N., Ericsson, M., Griffin, G.K., Dionne, D., Cuoco, M.S., Goder-Reiser, M.N., Sharova, T., Aguirre, A.J., et al. (2019). The enteric nervous system of the human and mouse colon at a single-cell resolution. bioRxiv, 746743.

Ema, M., Yokomizo, T., Wakamatsu, A., Terunuma, T., Yamamoto, M., and Takahashi, S. (2006). Primitive erythropoiesis from mesodermal precursors expressing VE-cadherin, PECAM-1, Tie2, endoglin, and CD34 in the mouse embryo. Blood 108, 4018-4024.

Espinosa-Medina, I., Jevans, B., Boismoreau, F., Chettouh, Z., Enomoto, H., Muller, T., Birchmeier, C., Burns, A.J., and Brunet, J.F. (2017). Dual origin of enteric neurons in vagal Schwann cell precursors and the sympathetic neural crest. Proc Natl Acad Sci U S A 114, 1198011985.

Freyer, L., Aggarwal, V., and Morrow, B.E. (2011). Dual embryonic origin of the mammalian otic vesicle forming the inner ear. Development 138, 5403-5414.

Gianino, S., Grider, J.R., Cresswell, J., Enomoto, H., and Heuckeroth, 
R.O. (2003). GDNF availability determines enteric neuron number by controlling precursor proliferation. Development 130, 2187-2198.

Habeck, M. (2003). VENT cells--a load of hot air? Drug Discov Today 8, 332-333

Haghverdi, L., Lun, A.T.L., Morgan, M.D., and Marioni, J.C. (2018). Batch effects in single-cell RNA-sequencing data are corrected by matching mutual nearest neighbors. Nat Biotechnol 36, 421-427.

Hao, M.M., and Young, H.M. (2009). Development of enteric neuron diversity. J Cell Mol Med 13, 1193-1210.

Herrick, S.E., and Mutsaers, S.E. (2004). Mesothelial progenitor cells and their potential in tissue engineering. Int J Biochem Cell Biol 36, 621-642.

Honig, G., Liou, A., Berger, M., German, M.S., and Tecott, L.H. (2010). Precise pattern of recombination in serotonergic and hypothalamic neurons in a Pdx1-cre transgenic mouse line. J Biomed Sci 17, 82.

Jarret, A., Jackson, R., Duizer, C., Healy, M.E., Zhao, J., Rone, J.M., Bielecki, P., Sefik, E., Roulis, M., Rice, T., et al. (2020). Enteric Nervous System-Derived IL-18 Orchestrates Mucosal Barrier Immunity. Cell 180, 50-63 e12.

Kawaguchi, M., Bader, D.M., and Wilm, B. (2007). Serosal mesothelium retains vasculogenic potential. Dev Dyn 236, 2973-2979.

Kim, M., Rosenbaum, C., Schlegel, N., Grumaz, C., Sohn, K., Isbert, C., Walles, H., and Metzger, M. (2019). Obstructed defecation-an enteric neuropathy? An exploratory study of patient samples. Int J Colorectal Dis 34, 193-196.

Klotz, L., Norman, S., Vieira, J.M., Masters, M., Rohling, M., Dube, K.N., Bollini, S., Matsuzaki, F., Carr, C.A., and Riley, P.R. (2015). Cardiac lymphatics are heterogeneous in origin and respond to injury. Nature 522, 62-67.

Kulkarni, S., Ganz, J., Bayrer, J., Becker, L., Bogunovic, M., and Rao, M. (2018). Advances in Enteric Neurobiology: The "Brain" in the Gut in Health and Disease. J Neurosci 38, 9346-9354.

Kulkarni, S., Micci, M.A., Leser, J., Shin, C., Tang, S.C., Fu, Y.Y., Liu, L., Li, Q., Saha, M., Li, C., et al. (2017). Adult enteric nervous system in health is maintained by a dynamic balance between neuronal apoptosis and neurogenesis. Proc Natl Acad Sci U S A 114, E3709-E3718.

Laranjeira, C., Sandgren, K., Kessaris, N., Richardson, W., Potocnik, A., Vanden Berghe, P., and Pachnis, V. (2011). Glial cells in the mouse enteric nervous system can undergo neurogenesis in response to injury. J Clin Invest 121, 3412-3424.

Lescroart, F., Chabab, S., Lin, X., Rulands, S., Paulissen, C., Rodolosse, A., Auer, H., Achouri, Y., Dubois, C., Bondue, A., et al. (2014). Early lineage restriction in temporally distinct populations of Mesp1 progenitors during mammalian heart development. Nat Cell Biol 16, 829-840.

Mao, Y., Wang, B., and Kunze, W. (2006). Characterization of myenteric sensory neurons in the mouse small intestine. J Neurophysiol 96, 998-1010.

Mizuno, S., Kurosawa, T., Matsumoto, K., Mizuno-Horikawa, Y., Okamoto, M., and Nakamura, T. (1998). Hepatocyte growth factor prevents renal fibrosis and dysfunction in a mouse model of chronic renal disease. J Clin Invest 101, 1827-1834.

Morarach, K., Mikhailova, A., Knoflach, V., Memic, F., Kumar, R., Li, W., Ernfors, P., and Marklund, U. (2020). Diversification of molecularly defined myenteric neuron classes revealed by single cell RNA-sequencing. bioRxiv, 2020.2003.2002.955757.

Natarajan, D., Marcos-Gutierrez, C., Pachnis, V., and de Graaff, E. (2002). Requirement of signalling by receptor tyrosine kinase RET for the directed migration of enteric nervous system progenitor cells during mammalian embryogenesis. Development 129, 5151-5160.

Obermayr, F., Hotta, R., Enomoto, H., and Young, H.M. (2013). Development and developmental disorders of the enteric nervous system. Nat Rev Gastroenterol Hepatol 10, 43-57.

Padovan-Merhar, O., Nair, G.P., Biaesch, A.G., Mayer, A., Scarfone,
S., Foley, S.W., Wu, A.R., Churchman, L.S., Singh, A., and Raj, A. (2015). Single mammalian cells compensate for differences in cellular volume and DNA copy number through independent global transcriptional mechanisms. Mol Cell 58, 339-352.

Papaconstantinou, J., Wang, C.Z., Zhang, M., Yang, S., Deford, J., Bulavin, D.V., and Ansari, N.H. (2015). Attenuation of p38alpha MAPK stress response signaling delays the in vivo aging of skeletal muscle myofibers and progenitor cells. Aging (Albany NY) 7 , 718-733.

Plaisance, I., Perruchoud, S., Fernandez-Tenorio, M., Gonzales, C., Ounzain, S., Ruchat, P., Nemir, M., Niggli, E., and Pedrazzini, T. (2016). Cardiomyocyte Lineage Specification in Adult Human Cardiac Precursor Cells Via Modulation of Enhancer-Associated Long Noncoding RNA Expression. JACC Basic TransI Sci 1, 472-493.

Prummel, K.D., Nieuwenhuize, S., and Mosimann, C. (2020). The lateral plate mesoderm. Development 147, dev175059.

Rappolee, D.A., lyer, A., and Patel, Y. (1996). Hepatocyte growth factor and its receptor are expressed in cardiac myocytes during early cardiogenesis. Circ Res 78, 1028-1036.

Rodrigues, D.M., Li, A.Y., Nair, D.G., and Blennerhassett, M.G. (2011). Glial cell line-derived neurotrophic factor is a key neurotrophin in the postnatal enteric nervous system. Neurogastroenterol Motil 23, e44-56.

Saffrey, M.J. (2014). Aging of the mammalian gastrointestinal tract: a complex organ system. Age (Dordr) 36, 9603.

Schafmayer, C., Harrison, J.W., Buch, S., Lange, C., Reichert, M.C., Hofer, P., Cossais, F., Kupcinskas, J., von Schönfels, W., Schniewind, B., et al. (2019). Genome-wide association analysis of diverticular disease points towards neuromuscular, connective tissue and epithelial pathomechanisms. Gut 68, 854.

Shenje, L.T., Andersen, P., Uosaki, H., Fernandez, L., Rainer, P.P., Cho, G.S., Lee, D.I., Zhong, W., Harvey, R.P., Kass, D.A., et al. (2014). Precardiac deletion of Numb and Numblike reveals renewal of cardiac progenitors. Elife 3, e02164.

Sohal, G.S., Ali, M.M., and Farooqui, F.A. (2002). A second source of precursor cells for the developing enteric nervous system and interstitial cells of Cajal. Int J Dev Neurosci 20, 619-626.

Sokoloff, A.J., Li, H., and Burkholder, T.J. (2007). Limited expression of slow tonic myosin heavy chain in human cranial muscles. Muscle Nerve 36, 183-189.

Stockdale, F.E., and Miller, J.B. (1987). The cellular basis of myosin heavy chain isoform expression during development of avian skeletal muscles. Dev Biol 123, 1-9.

Susaki, E.A., Tainaka, K., Perrin, D., Kishino, F., Tawara, T., Watanabe, T.M., Yokoyama, C., Onoe, H., Eguchi, M., Yamaguchi, S., et al. (2014). Whole-brain imaging with single-cell resolution using chemical cocktails and computational analysis. Cell 157, 726-739.

Taraviras, S., Marcos-Gutierrez, C.V., Durbec, P., Jani, H., Grigoriou, M., Sukumaran, M., Wang, L.C., Hynes, M., Raisman, G., and Pachnis, V. (1999). Signalling by the RET receptor tyrosine kinase and its role in the development of the mammalian enteric nervous system. Development 126, 2785-2797.

Uesaka, T., Nagashimada, M., and Enomoto, H. (2015). Neuronal Differentiation in Schwann Cell Lineage Underlies Postnatal Neurogenesis in the Enteric Nervous System. J Neurosci 35, 9879-9888.

Uesaka, T., Nagashimada, M., Yonemura, S., and Enomoto, H. (2008). Diminished Ret expression compromises neuronal survival in the colon and causes intestinal aganglionosis in mice. J Clin Invest 118, 1890-1898.

Uesaka, T., Young, H.M., Pachnis, V., and Enomoto, H. (2016). Development of the intrinsic and extrinsic innervation of the gut. Dev Biol 417, 158-167.

Wang, H., Hughes, I., Planer, W., Parsadanian, A., Grider, J.R., Vohra, B.P., Keller-Peck, C., and Heuckeroth, R.O. (2010). The timing and location of glial cell line-derived neurotrophic factor expression de- 
bioRxiv preprint doi: https://doi.org/10.1101/2020.08.25.262832; this version posted August 25, 2020. The copyright holder for this preprint (which was not certified by peer review) is the author/funder. All rights reserved. No reuse allowed without permission.

\section{BioRxiv Preprint}

termine enteric nervous system structure and function. J Neurosci 30, 1523-1538.

Wilm, B., Ipenberg, A., Hastie, N.D., Burch, J.B., and Bader, D.M. (2005). The serosal mesothelium is a major source of smooth muscle cells of the gut vasculature. Development 132, 5317-5328.

Xue, R., Gu, H., Quu, Y., Guo, Y., Korteweg, C., Huang, J., and Gu, J. (2016). Expression of Cystic Fibrosis Transmembrane Conductance Regulator in Ganglia of Human Gastrointestinal Tract. Sci Rep 6, 30926.

Young, H.M., Hearn, C.J., and Newgreen, D.F. (2000). Embryology and development of the enteric nervous system. Gut 47 Suppl 4, iv12-14; discussion iv26.

Young, H.M., and Newgreen, D. (2001a). Enteric neural crest-derived cells: Origin, identification, migration, and differentiation. The Anatomical Record 262, 1-15.

Young, H.M., and Newgreen, D. (2001b). Enteric neural crest-derived cells: origin, identification, migration, and differentiation. Anat Rec $262,1-15$.

Zeisel, A., Hochgerner, H., Lonnerberg, P., Johnsson, A., Memic, F., van der Zwan, J., Haring, M., Braun, E., Borm, L.E., La Manno, G., et al. (2018). Molecular Architecture of the Mouse Nervous System. Cell 174, 999-1014 e1022. 